\title{
A Stochastic Model for Adsorption Kinetics
}

\author{
Silvia Rodríguez-Narciso $\mathbb{D},{ }^{1}$ Juan Antonio Lozano-Álvarez $\mathbb{D},{ }^{2}$ Rogelio Salinas-Gutiérrez $\mathbb{D}{ }^{1}$ \\ and Netzahualcóyotl Castañeda-Leyva $\mathbb{i}^{1}$ \\ ${ }^{1}$ Departamento de Estadística, Universidad Autónoma de Aguascalientes, Av. Universidad 940, Ciudad Universitaria, Ags., \\ 20131 Aguascalientes, Mexico \\ ${ }^{2}$ Departamento de Ingeniería Bioquímica, Universidad Autónoma de Aguascalientes, Av. Universidad 940, Ciudad Universitaria, \\ Ags., 20131 Aguascalientes, Mexico
}

Correspondence should be addressed to Silvia Rodríguez-Narciso; srodrign@correo.uaa.mx

Received 20 January 2021; Revised 22 April 2021; Accepted 10 May 2021; Published 27 May 2021

Academic Editor: Adrián Bonilla-Petriciolet

Copyright (C) 2021 Silvia Rodríguez-Narciso et al. This is an open access article distributed under the Creative Commons Attribution License, which permits unrestricted use, distribution, and reproduction in any medium, provided the original work is properly cited.

\begin{abstract}
A novel stochastic model is proposed to characterize the adsorption kinetics of pollutants including dyes (direct red 80 and direct blue 1), fluoride ions, and cadmium ions removed by calcium pectinate (Pec-Ca), aluminum xanthanate (Xant- $\mathrm{Al})$, and reed leaves, respectively. The model is based on a transformation over time following the Ornstein-Uhlenbeck stochastic process, which explicitly includes the uncertainty involved in the adsorption process. The model includes stochastic versions of the pseudo-first-order (PFO), pseudo-second-order (PSO), and pseudo- $n$-order (PNO) models. It also allows the estimation of the adsorption parameters, including the maximum removal capacity $\left(q_{e}\right)$, the adsorption rate constant $\left(k_{n}\right)$, the reaction pseudoorder $(n)$, and the variability $\left(\sigma^{2}\right)$. The model fitted produced $R^{2}$ values similar to those of the nonstochastic versions of the PFO, PSO, and PNO models; however, the obtained values for each parameter indicate that the stochastic model better reproduces the experimental data. The $q_{e}$ values of the Pec-Ca-dye, Xant-Al-fluoride, and reed leaf-Cd ${ }^{+2}$ systems ranged from 2.0 to $9.7,0.41$ to 1.9 , and 0.04 and $0.29 \mathrm{mg} / \mathrm{g}$, respectively, whereas the values of $k_{n}$ ranged from 0.051 to $0.286,0.743$ to 75.73 , and 0.756 to $8.861(\mathrm{mg} / \mathrm{g})^{1-n} / \mathrm{min}$, respectively. These results suggest a variability in the parameters $q_{e}$ and $k_{n}$ inherent to the natures of the adsorbate and adsorbent. The obtained $n$ values ranged from 1.13 to 2.02 for the Pec-Ca-dye system, $1.0-3.5$ for the Xant-Al-fluoride system, and 1.8-3.8 for the reed leaf-Cd ${ }^{+2}$ system. These ranges indicate the flexibility of the stochastic model to obtain fractional $n$ values, resulting in high $R^{2}$ values. The variability in each system was evaluated based on $\sigma^{2}$. The developed model is the first to describe pollutant removal kinetics based on a stochastic differential equation.
\end{abstract}

\section{Introduction}

Water pollution is currently a transcendental issue. Numerous approaches including chemical, biological, and physical methods have been used to remove different contaminants that affect aquatic ecosystems. Among these methods, adsorption is relatively inexpensive and easy to scale up to industrial levels and shows great potential to remove specific pollutants from aqueous media [1-4].

Three main processes are involved in the adsorption of a compound present in an aqueous environment: (1) external diffusion, (2) intraparticle diffusion, and (3) surface reaction. However, under vigorous agitation, stages (1) and (2) have very little influence on the transport of contaminant from the aqueous phase to the inner surface of the adsorbent, and the limiting step in pollutant removal (i.e., the ratecontrolling step) is thought to be the binding between the adsorbate and adsorbent molecules at the liquid-solid interface [5]. In this case, several models have been used to describe the binding of chemical species contained in aqueous solution to the surface of an adsorbent material. The pseudo-first-order (PFO) model has been widely applied to the removal of different pollutants from aqueous media [6].

This model is described by the equation

$$
q_{t}=q_{e}\left(1-e^{-k_{1} t}\right), \quad t \geq 0,
$$


where $q_{t}$ is the adsorption capacity of the adsorbent material ( $\mathrm{mg} / \mathrm{g}$ ) at time $t(\mathrm{~min}), q_{e}$ is the maximum adsorption capacity when equilibrium is reached $(\mathrm{mg} / \mathrm{g})$, and $k_{1}$ is the PFO rate constant $(1 / \mathrm{min})$. Previously, in equation $(1), q_{e}$ is a parameter that must be known or estimated. However, this model does not accurately describe the experimental adsorption data in all cases. For some adsorption systems, the pseudo-second-order (PSO) model [7] is more appropriate:

$$
q_{t}=\frac{k_{2} q_{e}^{2} t}{1+k_{2} q_{e} t}, \quad t \geq 0
$$

where $q_{t}, q_{e}$, and $t$ are as defined above and $k_{2}$ is the PSO rate constant (g/mg min). Reference [8] showed that the PSO model best describes the experimental adsorption data when the initial adsorbate concentration in aqueous solution is low, suggesting an error related to the initial contaminant concentration in solution. Furthermore, when considering a large amount of experimental kinetic data for a system near equilibrium, the coefficient of determination suggests that the PSO model well fits the experimental data [9].

To model fractional-order kinetics, the pseudo- $n$-order (PNO) model [10-12] was developed as a general model:

$$
q_{t}=q_{e}\left(1-\frac{1}{\left(1+k_{n}(n-1) q_{e}^{n-1} t\right)^{1 /(n-1)}}\right), \quad t \geq 0
$$

where $k_{n}$ is the adsorption rate constant. The PNO model, which includes the PFO and PSO models, provides better fits to some experimental data compared to the PFO or PSO model [12-14]. Unlike the PFO and PSO models, the PNO model has the advantage of calculating the pseudoorder from the experimental kinetic data, and it is not necessary to assign a preestablished initial value to $q_{e}$.

However, based on the literature, no unified criteria for obtaining $q_{e}$ have been established among research groups. Furthermore, when considering the linear forms of these models, the coefficients of determination are higher than those obtained from their respective nonlinear forms; that is, the linear models overestimate the coefficients of determination. Therefore, the original nonlinear forms of the models are recommended [9].

In view of the above, in this work, the transformed Ornstein-Uhlenbeck (TOU) model is applied to characterize adsorption kinetics. The TOU model includes stochastic versions of the PFO, PSO, and PNO models. The OrnsteinUhlenbeck (OU) model has been applied in various areas such as finance, environmental modeling, and biological systems [15-18].

This novel TOU adsorption model explicitly includes the uncertainty (randomness) that exists in the adsorbate removal data and provides additional information that can be used to reduce the cost of adsorption-based pollutant removal. The TOU model combines the variability with an equilibrium point. Moreover, the proposed model does not require knowledge of $q_{e}$, and it is not necessary that kinetic measurements be made up until the equilibrium state is reached. The proposed model was applied in three adsorbent-adsorbate systems: direct red 80 (DR80) and direct blue 1 (DB1) dyes adsorbed by calcium pectinate (Pec-Ca); the removal of fluorides using aluminum xanthanate (Xant-Al); and cadmium removal by reed leaves.

\section{Materials and Methods}

2.1. Materials. Pectin from citrus peel, DB1, DR80, and xanthan were purchased from Sigma-Aldrich (St. Louis, MO, USA). Sodium hydroxide, calcium chloride dihydrate, hydrochloric acid, sodium chloride, and distilled water were obtained from J.T. Baker (México State, México). Aluminum chloride hexahydrate was provided by Golden Bell (México City, México).

\subsection{Experiments}

2.2.1. Synthesis of Pec-Ca. The synthesis of Pec-Ca was performed according to [19] with some modifications. Pectin was dissolved in a basic aqueous solution $(\mathrm{pH}=12.0)$ for a period of $8 \mathrm{~h}\left(28^{\circ} \mathrm{C}\right)$ to obtain maximum hydration. Subsequently, the resulting solution $(1 \% w / v)$ was adjusted to $\mathrm{pH}$ 7.0. An aliquot of calcium chloride dihydrate $(5 \% w / v)$ was added to this solution, and the resulting $\mathrm{Pec}-\mathrm{Ca}$ gel was allowed to stand at room temperature $\left(28^{\circ} \mathrm{C}\right.$ for $\left.24 \mathrm{~h}\right)$ to stop its consolidation. The resulting product was washed at least five times with distilled water to eliminate excess calcium ions in the gel. The gel was lyophilized and ground using a mortar. The obtained particulate was sieved to produce a powder with an average size of $0.297 \mathrm{~mm}$.

2.2.2. Dye Adsorption Kinetics. We experimentally studied the effect of $\mathrm{pH}$ on the removal efficiency of the two dyes. The experiments were carried out in triplicate, and the results are shown in the supplemental Figure S1. The optimal $\mathrm{pH}$ values for DR80 and DB1 removal were 3 and 2, respectively. Next, for each dye, a $500 \mathrm{~mL}$ aliquot of the aqueous dye solution that had been adjusted to the optimal $\mathrm{pH}$ was placed in a $2 \mathrm{~L}$ Erlenmeyer flask. After measuring the initial absorbance (at $533 \mathrm{~nm}$ for DR80 and $618 \mathrm{~nm}$ for DB1) at $t=0 \mathrm{~min}$ using a UV-visible spectrophotometer (Evolution 201, Thermo Scientific), $1.0 \mathrm{~g}$ of Pec-Ca was added to the aqueous dye solution followed by orbital shaking (100 rpm). After a determined time, a small amount of the aqueous dye solution was centrifuged at $17,000 \mathrm{rpm}$ for $5 \mathrm{~min}$, and the absorbance of the supernatant was measured at the defined wavelength. The dye concentration at each time point was obtained by interpolating the absorbance value using the standard curve constructed for each dye. The value of $q_{t}(\mathrm{mg} / \mathrm{g})$ for each sample was determined according to the following equation:

$$
q_{t}=\left(C_{0}-C_{f}\right) \times \frac{V}{m}
$$

where $C_{0}$ and $C_{f}$ correspond to the initial and final dye concentrations $(\mathrm{mg} / \mathrm{L})$, respectively; $V$ is the volume of dye solution (L); and $m$ is the mass of adsorbent (g). All dye adsorption kinetic experiments were carried out in triplicate, 
with the three replicates termed experiment 1 , experiment 2, and experiment 3. It is important to mention that the estimated kinetic parameters are based on the integrated data obtained from the three experiments. This approach is more convenient than the typical method in which the model is fitted using the average data. However, this procedure underestimates the experimental error.

2.2.3. Synthesis of Xant-Al. Xant-Al was synthesized according to [20] except that no dye was included in the synthetic process. An aqueous solution of xanthan $(1 \% w / v)$ was obtained hydrating $5 \mathrm{~g}$ of this polysaccharide in $500 \mathrm{~mL}$ of distilled water at $28^{\circ} \mathrm{C}$ for $24 \mathrm{~h}$. An equivalent volume of $\mathrm{AlCl}_{3} 6 \mathrm{H}_{2} \mathrm{O}$ solution $(5 \% \mathrm{w} / \mathrm{v})$ was added to the xanthan dispersion, resulting in the formation of a gel (Xant-Al), which was kept at room temperature $\left(28^{\circ} \mathrm{C}\right)$ for $24 \mathrm{~h}$ to complete gel formation. The subsequent treatment applied to the obtained gel to produce the powder form of Xant-Al was similar to the procedure used for $\mathrm{Pec}-\mathrm{Ca}$ (see the previous section).

2.2.4. Fluoride Adsorption Kinetics. A $1 \mathrm{~L}$ aliquot of aqueous fluoride solution with the $\mathrm{pH}$ adjusted to the optimal value for fluoride removal was placed in a $2.8 \mathrm{~L}$ Fernbach flask. A sample was taken to analyze the initial fluoride concentration at $t=0$. Subsequently, $2.0 \mathrm{~g}$ of Xant-Al was added to the aqueous fluoride solution followed by simultaneous incubation at $28^{\circ} \mathrm{C}$ and orbital shaking $(100 \mathrm{rpm})$. Samples were taken at different time points and centrifuged at 17,000 rpm for $1 \mathrm{~min}$. The fluoride content in the supernatant was then determined using an ion-selective electrode [21]. Finally, the $q_{t}$ value for each sample was calculated in accordance with equation (4). All kinetic measurements were carried out in triplicate, and the replicates were named as described in the previous section.

2.2.5. Data from Other Sources. Kinetic data for cadmium adsorption on reed leaves previously reported by [22] were used in conjunction with the kinetic data from the dye and fluoride removal experiments to construct the TOU model, as detailed in the next section.

\section{Modeling}

The study of adsorption kinetics and identification of a model that properly describes the experimental kinetic data represent a valuable strategy for studying pollutant removal from different effluents. As mentioned above, the PFO and PSO models are the most used models for describing adsorption kinetics because their linear forms provide adequate fits to different systems with high coefficients of determination and simplicity. On the other hand, the PNO model, which includes the PFO and PSO models, is applied to fractionalorder adsorption kinetics. While all these models can describe the kinetics in specific cases, they do not allow the inclusion of a variance component.

In contrast, in this study, a TOU model is proposed that allows explaining and predicting removal kinetics. This model includes a term that represents the randomness of the adsorption process. This approach offers advantages over the classical versions of the aforementioned models. As a transformation of the OU model, the TOU model combines the variability with an equilibrium state point [15]. The OU model is a continuous-time, Gaussian, and asymptotically stationary model [23].

Notably, the TOU model contains stochastic versions of the PFO, PSO, and PNO models. Thus, the TOU model provides a general approach for modeling adsorption kinetics, including the randomness of the adsorption process. This means that the application of the TOU model is not limited to the stochastic version of the PNO model. For instance, the TOU model can be applied to fixed pseudo- $n$-order kinetics, as for the classical PFO and PSO models.

The removal kinetics are characterized by the parameters of the TOU model, including the maximum removal $q_{e}$, the pseudoorder $n$, the rate constant $k_{n}$, and $\sigma^{2}$, which represents the randomness of the adsorption process.

To introduce the TOU model, the stochastic version of the PFO model (1) is first developed, and the relationship between the ordinary and stochastic versions is described.

The PFO model (1) obeys the ordinary differential equation

$$
d q_{t}=k_{1}\left(q_{e}-q_{t}\right) d t
$$

The kinetics $\left\{q_{t}\right\}_{t \geq 0}$ can be considered to behave in accordance with the OU model, which obeys the stochastic differential equation (SDE)

$$
d q_{t}=\lambda\left(q_{e}-q_{t}\right) d t+\sigma d W_{t}
$$

where $\lambda$ is a rate constant and $q_{e}$ is the maximum removal capacity, which is described in (1). The error term $\sigma d W_{t}$ has a normal distribution with a zero mean and variance $\sigma^{2} d t$, where $\left\{W_{t}\right\}_{t \geq 0}$ denotes a standard Brownian motion and $\sigma>0$. Hence, the error term takes both positive and negative values.

A distinction must be drawn between the ordinary differential equation (5) and the SDE (6). The latter considers the adsorption-desorption process to be a stochastic phenomenon with a chaotic contribution arising from the dynamic nature of the process. Other sources of noise include the heterogeneity of the material used as an adsorbent and the measurement process. In general, the parameter $\sigma$ includes all these sources of noise.

The solution of the SDE (6) is

$$
q_{t}=q_{0} e^{-\lambda t}+q_{e}\left(1-e^{-\lambda t}\right)+\sigma \int_{0}^{t} e^{-\lambda(t-s)} d W_{s}
$$

where $q_{0} \geq 0$ is the kinetics value at time $t=0$. Typically, $q_{0}$ is assumed to be a known constant. An advantage of the model (6) is its functionality for any value of $q_{0}$. When $q_{0}>0$, it means that the adsorbents have been partially used (i.e., they have the capacity to continue removing the adsorbate from the polluted aqueous medium). For an adsorption process, $q_{0}$ is considered to be equal to 0 , and this is assumed hereafter. 
The solution of the OU model (6) is simplified as

$$
q_{t}=q_{e}\left(1-e^{-\lambda t}\right)+\sigma \int_{0}^{t} e^{-\lambda(t-s)} d W_{s} .
$$

Based on the properties of standard Brownian motion, $q_{t}$ is a random variable with a normal distribution and mean

$$
E\left[q_{t}\right]=q_{e}\left(1-e^{-\lambda t}\right)
$$

An increasing global trend in adsorption is expected. The variance of $q_{t}$ is given by

$$
V\left[q_{t}\right]=\sigma^{2} \frac{1-e^{-2 \lambda t}}{2 \lambda}=\tau^{2}\left(1-e^{-2 \lambda t}\right), \quad t \geq 0,
$$

where $\tau^{2}=\left(\sigma^{2} /(2 \lambda)\right)>0$. The long-term kinetics also have a normal distribution with mean $q_{e}$ and variance $\tau^{2}$. In this way, $q_{e}$ is the mean maximum capacity of adsorption when the equilibrium state is reached, while the maximum variance of the adsorption kinetics is $\tau^{2}$.

Note that the mean function $E\left[q_{t}\right]$ (9) solves the ordinary differential equation (5), with $\lambda=k_{1}$. In this sense, with the proposed stochastic approach, the PFO model is reproduced in terms of the mean adsorption kinetics (9). The variability component in expression (8) explains the dispersion of the adsorption process around its mean. The natural uncertainty of the adsorption process is represented by expression (10).

As mentioned above, researchers that use the PFO model widely assume a fixed $q_{e}$ value that is restricted to be greater than the maximum experimental adsorption result. However, this assumption tends to overestimate the value of $q_{e}$, which limits the ability to estimate the model parameters and the corresponding predict adsorption.

In contrast, using the approach proposed for model (8), no previous information about the value of $q_{e}$ is required. Rather, this parameter is estimated as part of the proposed method.

Now, the proposed TOU model is described. The TOU model is a generalization of the OU kinetic adsorption model (8).

The model proposed for the adsorption kinetics of different pollutants in aqueous media is a time transformation of the OU model (8). In this sense, consider that the adsorption process $\left\{q_{t}\right\}_{t \geq 0}$ comes from the following time transformation:

$$
q_{t}=X_{g(t)}
$$

where $\left\{X_{t}\right\}_{t \geq 0}$ represents an OU model (8) (with parameters $\left.q_{e}, \lambda, \tau>0\right)$ and $g(t)$ is a strictly increasing function with $g(0)=0$ and $g(\infty)=\lim _{t \longrightarrow \infty} g(t)=\infty$. This transformation implies

$$
\begin{aligned}
q_{0} & =X_{g(0)}=X_{0}=0, \\
E\left[q_{t}\right] & =E\left[X_{g(t)}\right]=q_{e}\left(1-e^{-\lambda g(t)}\right), \\
V\left[q_{t}\right] & =\mathrm{V}\left[X_{g(t)}\right]=\tau^{2}\left(1-e^{-2 \lambda g(t)}\right),
\end{aligned}
$$

and

$$
\begin{aligned}
& \lim _{t \rightarrow \infty} E\left[q_{t}\right]=\lim _{t \rightarrow \infty} E\left[X_{g(t)}\right]=\lim _{t \longrightarrow \infty}\left[q_{e}\left(1-e^{-\lambda g(t)}\right)\right]=q_{e}, \\
& \lim _{t \rightarrow \infty} V\left[q_{t}\right]=\lim _{t \rightarrow \infty} V\left[X_{g(t)}\right]=\lim _{t \longrightarrow \infty}\left[\tau^{2}\left(1-e^{-2 \lambda g(t)}\right)\right]=\tau^{2} .
\end{aligned}
$$

The time transformation preserves the role of the parameters $q_{e}, \lambda$, and $\tau$. This means that the properties that characterize the OU model in terms of its parameters $q_{e}, \lambda$, and $\tau$ are inherited by the new kinetic model $q_{t}$. For instance, the parameter $q_{e}$ is the maximum adsorption value found in the equilibrium state, both for $q_{t}$ and $X_{t}$. A similar interpretation holds for the parameter $\tau^{2}$, which represents the asymptotic variance and maximum variance for both processes. In expression (24) introduced later, the common role of the parameter $\lambda$ is verified for both processes.

The time transformation $g(t)$ has a flexible effect that explains a wide variety of adsorption kinetics, preserving the properties of equilibrium and uncertainty from the underlying OU model.

Specifically, the proposed family of transformations in the time domain is given by

$$
g(t)=\frac{1}{a} \ln (1+a t), \quad t \geq 0
$$

with $a>0$. The OU model (without transformation) represents the case $a=0$, which corresponds to the limit when $a \longrightarrow 0$ :

$$
g(t)=t, \quad t \geq 0
$$

Applying the time transformation given in equation (14) to expression (11), the proposed TOU model is

$$
\begin{aligned}
q_{t} & =X_{g(t)}=q_{e}\left(1-e^{-\lambda g(t)}\right)+\sigma \int_{0}^{g(t)} e^{-\lambda(g(t)-s)} d W_{s} \\
& =q_{e}\left(1-e^{-(\lambda / a) \ln (1+a t)}\right)+\sigma \int_{0}^{g(t)} e^{-\lambda(g(t)-s)} d W_{s} \\
& =q_{e}\left(1-\frac{1}{(1+a t)^{\lambda / a}}\right)+\sigma \int_{0}^{g(t)} e^{-\lambda(g(t)-s)} d W_{s} .
\end{aligned}
$$


Based on the properties of the OU model, $q_{t}$ is a normal random variable with mean defined by

$$
E\left[q_{t}\right]=E\left[X_{g(t)}\right]=q_{e}\left(1-\frac{1}{(1+a t)^{\lambda / a}}\right)
$$

and variance determined by

$$
V\left[q_{t}\right]=V\left[X_{g(t)}\right]=\tau^{2}\left(1-\frac{1}{(1+a t)^{2 \lambda / a}}\right) .
$$

The TOU model parameters are $q_{e}, \lambda, \tau$, and $a$. The new parameter $a$ is related to the reaction order $n$ of the kinetic process, as explained here:

$$
n=\frac{a}{\lambda}+1=\frac{\lambda+a}{\lambda} .
$$

Expression (17) can be converted into

$$
E\left[q_{t}\right]=q_{e}\left(1-\frac{1}{\left(1+k_{n}(n-1) q_{e}^{n-1} t\right)^{1 /(n-1)}}\right),
$$

where

$$
k_{n}=\frac{\lambda}{q_{e}^{n-1}} .
$$

Therefore, the mean function (20) of the TOU model matches the PNO model (3). In this sense, the PNO model is a particular case of the TOU model, whenever $\sigma=0$. The interpretation of the parameters $q_{e}, n$, and $k_{n}$ is the same in both models.

The relationship between the TOU and PNO models is also apparent when considering the rate function of the proposed TOU model, which is derived from expression (20):

$$
\frac{d}{d t} E\left[q_{t}\right]=\frac{q_{e} \lambda}{(1+a t)^{(\lambda+a) / a}}=k_{n}\left(q_{e}-E\left[q_{t}\right]\right)^{n} .
$$

Expression (3), which satisfies the ordinary differential equation of the PNO model, was reported by [10].

The proposed TOU model (16) has the advantage of including a variability component, which explains the dispersion of the kinetics around the mean function (20). In this way, from expressions (18)-(21), the natural uncertainty in the adsorption process is represented as the variance

$$
V\left[q_{t}\right]=\tau^{2}\left(1-\frac{1}{\left(1+k_{n}(n-1) q_{e}^{n-1} t\right)^{2 /(n-1)}}\right) .
$$

In the case without the time transformation (i.e., when $a=0$ and $n=1$ ), the stochastic version of the PFO model (1) is obtained. In addition, the stochastic version of the PSO model (2) is obtained with $a=\lambda$ and $n=2$. In general terms, the TOU model includes the stochastic versions of the fractional-order models. The relationship between the parameters $n$ and $a$ is given by the direct linear equation (19); that is, $a=(n-1) \lambda$. The kinetic curvature depends on the parameter $n$ or, equivalently, $a$ (see Section 2.3 in [12].

Here, the interpretation of some parameters of the TOU model is discussed. When $t=0$ in equation (22), the initial kinetic rate is given by

$$
\frac{d}{d t} E\left[q_{t}\right]_{t=0}=q_{e} \lambda .
$$

On the right side of expression (24), the initial kinetic rate depends on the parameters $q_{e}$ and $\lambda$. In this sense, the greater the equilibrium level $q_{e}$, the greater the initial kinetic rate. A similar conclusion applies for $\lambda$. Together with $q_{e}$, the parameter $\lambda$ appears in all kinetics of order $n$. Both parameters are comparable between kinetics of several orders. Therefore, the reaction order $n$ does not determine the initial rate, given by expression (24). Lastly, $k_{n}$ is the rate constant of pseudoorder $n$.

In adsorption kinetics, another important parameter is the time needed to reach half of the $q_{e}$ value, which is denoted herein as $t_{1 / 2}$. For the stochastic version of the PFO model $(n=1, a=0)$, this time satisfies the following equation:

$$
E\left[q_{t_{1 / 2}}\right]=q_{e}\left(1-e^{-\lambda t_{1 / 2}}\right)=\frac{q_{e}}{2} .
$$

Based on this equation, we have

$$
t_{1 / 2}=\frac{\ln 2}{\lambda} .
$$

Analogously, for the stochastic model of order $n>1$ $(a>0)$, the mean time is

$$
t_{1 / 2}=\frac{2^{a / \lambda}-1}{a}=\frac{2^{n-1}-1}{\lambda(n-1)} .
$$

On the other hand, with the stochastic approach, the information obtained from repeated experiments is integrated to obtain a single estimation of the removal kinetic parameters. The model can predict adsorption at any time $t>0$. In particular, long-term predictions are obtained with the estimated value of $q_{e}$.

3.1. Determination of the Time $t_{e}$ Required to Reach the Equilibrium State. An additional advantage of the TOU model is that it estimates the distribution of time $t_{e}$ for attaining the maximum adsorption level in the equilibrium state $q_{e}$. In the process of adsorption, the removal of the pollutant tends to increase gradually until it becomes stable in the equilibrium state. The time $t_{e}$ needed to reach the equilibrium state is a continuous positive random variable, and its probability distribution is explicitly quantified $[15,24]$. The random variable $t_{e}$ is the time lapse in which the maximum amount of adsorbate $q_{e}$ is removed [25]. Its variability is attributed to the nature of the experiment and the amounts of both the adsorbate and adsorbent. The sources of noise 
also include the porosity of the adsorbent, which is related to its surface area; the temperature, $\mathrm{pH}$, ionic strength, polarity, and viscosity of the solution that contains the adsorbate; and the agitation speed. This means that when a specific adsorption experiment is repeated under the same conditions, the time at which the $q_{e}$ value is reached differs among the experimental replicates.

In formal terms, the random variable $t_{e}$ is defined as

$$
t_{e}=\inf \left\{t \geq 0: q_{t}=q_{e}\right\}
$$

For $n=1(a=0)$, the distribution and density functions of $t_{e}$ are given by

$$
F_{1}(t)=P\left(t_{e} \leq t\right)=2 \Phi\left(-\frac{q_{e}}{\tau \sqrt{e^{2 \lambda t}-1}}\right)
$$

and

$$
f_{1}(t)=F_{1}^{\prime}(t)=\frac{2 q_{e} \lambda}{\tau} \phi\left(-\frac{q_{e}}{\tau \sqrt{e^{2 \lambda t}-1}}\right) \frac{e^{2 \lambda t}}{\left(e^{2 \lambda t}-1\right)^{3 / 2}},
$$

where $\Phi(\cdot)$ and $\phi(\cdot)$ are the standard normal distribution and density functions $N(0,1)$, respectively. The subindex in $F_{1}(\cdot)$ and $f_{1}(\cdot)$ refers to case $n=1$.

The density function $f_{1}(t)$ is skewed to the right; thus, its median is recommended as a central measure:

$$
\operatorname{Median}_{1}=\frac{1}{2 \lambda} \ln \left(1+\left(\frac{q_{e}}{\tau \Phi^{-1}(3 / 4)}\right)^{2}\right)>0
$$

As for the case of $n>1(a>0)$, the distribution of $t_{e}$ is obtained from the corresponding distribution of the time required to reach equilibrium for the case $n=1$ by using the random variable transformation theorem. In this way, the distribution function for $t_{e}$ is

$$
\begin{aligned}
F_{n}(t) & =F_{1}\left(\frac{\ln (1+a t)}{a}\right) \\
& =2 \Phi\left(-\frac{q_{e}}{\tau \sqrt{(1+\lambda(n-1) t)^{2 / n-1}-1}}\right) .
\end{aligned}
$$

The corresponding density function is

$$
\begin{aligned}
f_{n}(t)= & \frac{1}{1+a t} f_{1}\left(\frac{\ln (1+a t)}{a}\right) \\
= & \frac{2 q_{e} \lambda}{\tau} \phi\left(-\frac{q_{e}}{\tau \sqrt{(1+\lambda(n-1) t)^{2 / n-1}-1}}\right) \\
& \times \frac{(1+\lambda(n-1) t)^{(3-n) / n-1}}{\left((1+\lambda(n-1) t)^{2 / n-1}-1\right)^{3 / 2}} .
\end{aligned}
$$

Given that the function $f_{n}(t)$ is not symmetrical, the median is a good measure of its central trend. In this case, the median of this distribution is defined by

$$
\text { Median }=\frac{e^{a \cdot \text { median }_{1}}-1}{a}=\frac{e^{\lambda(n-1) \text { median }_{1}}-1}{\lambda(n-1)},
$$

where median $_{1}$ refers to the median of the case $n=1$ (31).

Figure 1 shows a histogram of the time required to reach the equilibrium state obtained from the simulation of 1000 adsorption kinetics. To quantify the distribution of time needed to reach the equilibrium state based on the simulations, the corresponding theoretical density of the time required to reach equilibrium is also included in this figure. As shown in Figure 1, the $t_{e}$ value is not the same for all the simulated kinetics; therefore, it is necessary to propose an adequate estimation. Here, we propose using the median to estimate the time required to reach equilibrium.

The knowledge of the density function provides valuable information as it allows identifying the time range in which the adsorption kinetics reaches the $q_{e}$ value. It also facilitates the planning of experiments in sequence (i.e., the quality of prediction is assessed at each observation of adsorption kinetics). In this way, the experiment may be stopped before originally planned when the estimated distribution of $t_{e}$ does not change significantly with additional experimental runs, thereby reducing cost. For sequential techniques, see $[26,27]$ and references therein.

3.2. TOU Model Estimation. The adsorption parameters are estimated using the maximum likelihood method [28], which involves finding the model parameters that maximize the probability of reproducing the empirically observed data. In this case, the function to be maximized is a likelihood function whose arguments are the parameters of the model. To select the best TOU model for the experimental data, the likelihood function is maximized over the parameters $\lambda, q_{e}, a$, and $\tau$. Using the proposed approach, the fitted model for the optimal estimated parameters provides a high coefficient of determination $R^{2}$ (close to one).

As a first step, the likelihood function is obtained. Considering the properties of the TOU model, for $h, t \geq 0$, the increase in adsorption $q_{t+h}-q_{t}$ in a time interval with length $h$ has a normal distribution:

$$
\begin{aligned}
{\left[q_{t+h}-q_{t} \mid q_{t}\right] \sim \mathrm{N}(\text { mean }} & =\left(q_{e}-q_{t}\right)\left(1-e^{-\lambda(g(t+h)-g(t))}\right), \\
\text { variance } & \left.=\tau^{2}\left(1-e^{-2 \lambda(g(t+h)-g(t))}\right)\right),
\end{aligned}
$$

where $g(t)$ is the function defined in equation (14). Now, assuming that the process of adsorption is observed $N+1$ times,

$$
0 \leq t_{0}<t_{1}<\cdots<t_{N}
$$




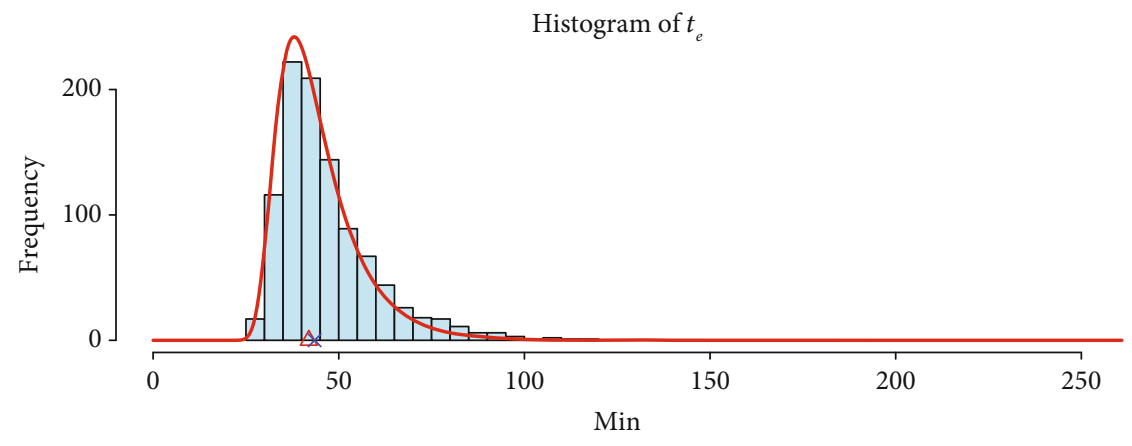

Sample size $=1000$

— Density function

Median

$\triangle$ Actual $=41.94 \mathrm{~min}$

$\times$ Sample $=43.5 \mathrm{~min}$

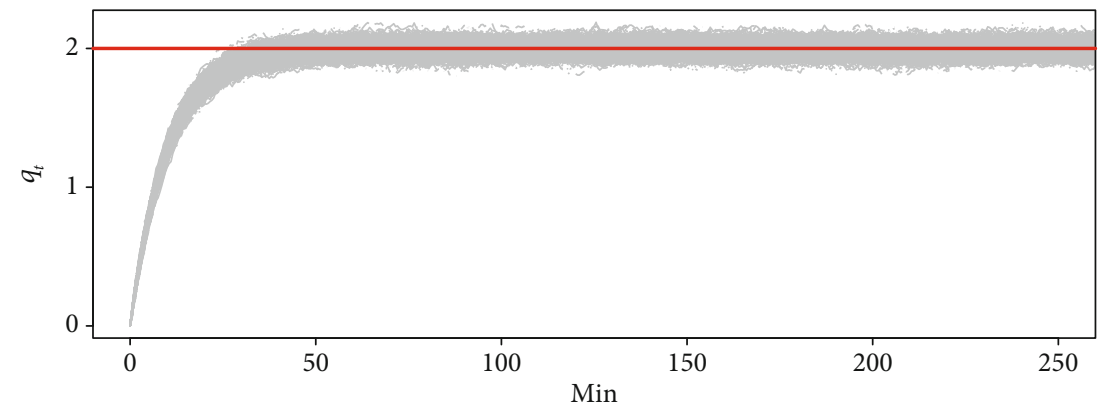

Sample size $=1000$

- Simulated kinetics

FIgURE 1: Adsorption kinetics obtained based on computer simulation (bottom) and the empirical distribution of the time required to reach the equilibrium state (top). The parameters used in the simulation are $q_{e}=2.0, n=1, k_{n}=0.1$, and $\sigma=0.02$.

where the successive increments of adsorption are given by

$$
\begin{aligned}
& z_{1}=q_{t_{1}}, \\
& z_{2}=q_{t_{2}}-q_{t_{1}}, \cdots, z_{N}=q_{t_{N}}-q_{t_{N-1}} .
\end{aligned}
$$

Based on equation (35) and the Markov property of the OU model, the joint density function of the increase vector is given by

$$
\begin{aligned}
f\left(z_{1}, \cdots, z_{N}\right) & \\
= & \prod_{i=1}^{N} \frac{1}{\tau \sqrt{2 \pi\left(1-e^{-2 \lambda h_{i}}\right)}} e^{-\left[z_{i}-\left(q_{e}-q_{t_{i-1}}\right)\left(1-e^{-\lambda h_{i}}\right)\right]^{2} /\left[2 \tau^{2}\left(1-e^{-2 \lambda h_{i}}\right)\right]} \\
& \propto \tau^{-N} \prod_{i=1}^{N}\left(1-e^{-2 \lambda h_{i}}\right)^{-1 / 2} \\
& \cdot e^{-\sum_{i=1}^{N}\left[z_{i}-\left(q_{e}-q_{t_{i-1}}\right)\left(1-e^{-\lambda h_{i}}\right)\right]^{2} /\left[2 \tau^{2}\left(1-e^{-2 \lambda h_{i}}\right)\right]},
\end{aligned}
$$

where $h_{i}=h_{i}(a)=g\left(t_{i}\right)-g\left(t_{i-1}\right)>0$ for $i=1, \cdots, N$. The likelihood function is then given as

$$
\begin{aligned}
& L\left(q_{e}, \lambda, a, \tau\right) \\
& =\tau^{-N} \prod_{i=1}^{N}\left(1-e^{-2 \lambda h_{i}}\right)^{-1 / 2} \\
& \quad \cdot e^{-\sum_{i=1}^{N}\left[z_{i}-\left(q_{e}-q_{t_{i-1}}\right)\left(1-e^{-\lambda h_{i}}\right)\right]^{2} /\left[2 \tau^{2}\left(1-e^{-2 \lambda h_{i}}\right)\right]},
\end{aligned}
$$

with parametric space $q_{e}, \lambda, \tau>0$, and $a \geq 0$. The corresponding log-likelihood function is

$$
\begin{aligned}
l\left(q_{e}, \lambda, a, \tau\right)= & -N \ln \tau-\frac{1}{2} \sum_{i=1}^{N} \ln \left(1-e^{-2 \lambda h_{i}}\right) \\
& -\frac{1}{2 \tau^{2}} \sum_{i=1}^{N} \frac{\left(z_{i}-\left(q_{e}-q_{t_{i-1}}\right)\left(1-e^{-\lambda h_{i}}\right)\right)^{2}}{1-e^{-2 \lambda h_{i}}} .
\end{aligned}
$$



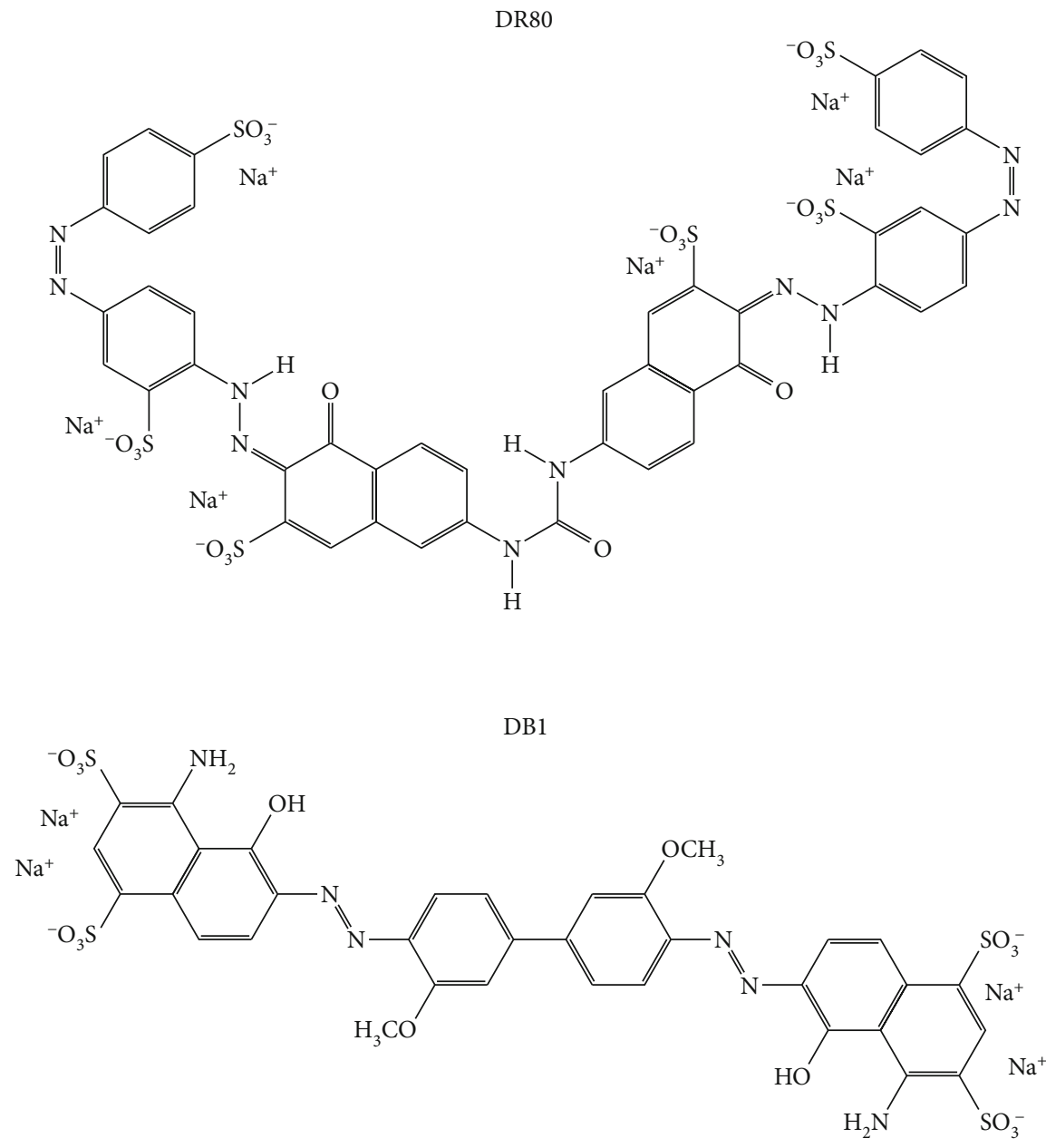

FIgure 2: Chemical structures of the dyes used in this study.

If there are $M$ repetitions of the adsorption experiment with observations made at the same time points, the loglikelihood function is

$$
\begin{aligned}
l\left(q_{e}, \lambda, a, \tau\right)= & -M N \ln \tau-\frac{M}{2} \sum_{i=1}^{N} \ln \left(1-e^{-2 \lambda h_{i}}\right) \\
& -\frac{1}{2 \tau^{2}} \sum_{j=1}^{M} \sum_{i=1}^{N} \frac{\left(z_{i j}-\left(q_{e}-q_{t_{i-1}, j}\right)\left(1-e^{-\lambda h_{i}}\right)\right)^{2}}{1-e^{-2 \lambda h_{i}}},
\end{aligned}
$$

for $q_{e}, \lambda, \tau>0$, and $a \geq 0$. The maximum likelihood estimator is the vector $\left(\widehat{q}_{e}, \widehat{\lambda}, \widehat{a}, \widehat{\tau}\right)$ that maximizes the function $l\left(q_{e}, \lambda\right.$, $a, \tau)$. The estimates of $n$ and $k_{n}$ are obtained upon substituting $\widehat{q}_{e}, \widehat{k}_{1}$, and $\widehat{a}$ into expressions (19) and (21), respectively.

Due to the complexity of the log-likelihood function, it is not possible to obtain analytical expressions for the maximum likelihood estimators. Thus, the Newton-Raphson method is used to obtain estimates of these parameters. For this purpose, a program was created using the statistics software R [29].

\section{Results and Discussion}

4.1. Dye Removal by Pec-Ca. Recently, various biomaterials and their derivatives have been used to remove different pollutants from aqueous media. In the case of dye removal, biopolymers such as chitin, chitosan, xanthan, alginic acid, and pectin have been applied [20,30-35]. In this study, Pec-Ca synthesized in our laboratory was used to remove DR80 and DB1 dyes, whose structures are shown in Figure 2. Hereafter, these systems are denominated as PecCa-DR80 and Pec-Ca-DB1, respectively.

The adsorption kinetics of dye removal were studied using different dye concentrations $(5,10,15$, and $10 \mathrm{mg} / \mathrm{L})$. All experiments were carried out in triplicate to ensure reliability, repeatability, and precision in the information obtained. The results obtained using all concentration values were included in developing the TOU model proposed in this article.

Figure 3 depicts the experimental kinetics of DR80 removal by Pec-Ca along with the theoretical curve obtained by applying the TOU model to the data obtained at different DR80 concentrations $(5-20 \mathrm{mg} / \mathrm{L})$. The TOU model provides an adequate fit to the experimental data for all concentration levels. On the other hand, $q_{t}$ increases drastically from 0 to 


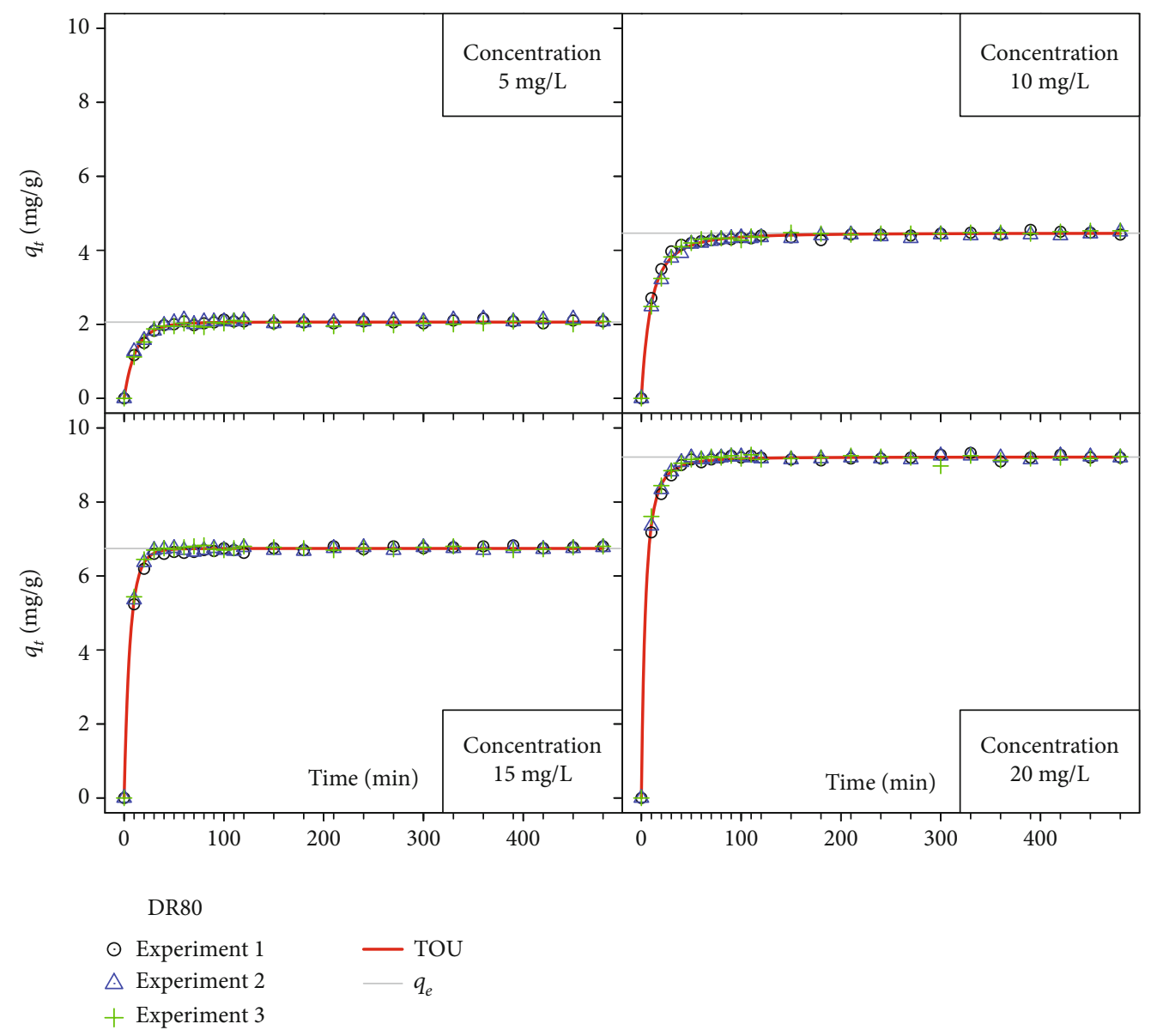

Figure 3: Experimental adsorption kinetics of DR80 dye removal by Pec-Ca for different dye concentrations and theoretical curves obtained by applying the TOU model to the experimental data.

40 min. Subsequently, $q_{t}$ decreases until the system reaches equilibrium (at approximately $200 \mathrm{~min}$ ).

Table 1 gives the estimated parameters obtained from the TOU model and the goodness-of-fit measures of this model when applied to the Pec-Ca-DR80 and Pec-Ca-DB1 systems. As indicated by the high coefficient of determination $\left(R^{2}\right)$ values (0.987-0.998), the low mean squared error (MSE) values (0.023-0.054), and the normalized standard deviation $(\Delta q)$ values $(0.866 \%-2.867 \%)$ in Table 1 , the TOU model provides adequate fits to the four experimental adsorption kinetics of DR80 by Pec-Ca. In addition, the estimated value of the maximum adsorption capacity at equilibrium $\left(q_{e}\right)$ tends to increase as the initial DR80 concentration increases.

For comparison with the proposed TOU model, Table 2 shows the kinetic parameters obtained using the PFO, PSO, and PNO kinetic equations for the Pec-Ca-DR80 and PecCa-DB1 systems. The coefficients of determination and values of $q_{e}, n$, and $k_{n}$ provided by the PNO model are similar to those obtained with the TOU model (see Tables 1 and 2). Thus, the TOU model is competitive with the PFO, PSO, and PNO models in terms of its ability to fit the experimental data.

The proposed TOU model gives the distribution of the time $t_{e}$ required to reach the equilibrium state, which is not possible with the other models (PFO, PSO, and PNO). Moreover, the TOU model provides an explicit estimate for the spread of the kinetics over the parameter $\sigma$.

Figure 4 shows the density functions of the time $t_{e}$ required to reach $q_{e}$. In most cases, $q_{e}$ is reached within the time interval from 40 to $200 \mathrm{~min}$. The medians of the density functions have similar magnitudes at different dye concentrations; however, the density is greater in the Pec-Ca-DB1 system than in the Pec-Ca-DR80 system because the value of $n$ is greater in the Pec-Ca-DB1 system, as discussed later.

On the other hand, the values of $n$ and $k_{n}$ for the Pec-CaDR80 system remain on the same order of magnitude (Table 1). Thus, the $n$ values resulting from the TOU model are between 1.137 and 1.48, while $k_{n}$ ranges from 0.079 to $0.135 \mathrm{~min}^{-1} \cdot(\mathrm{mg} / \mathrm{g})^{1-n}$. In previous reports, the PSO and PNO models include the following assumptions [36]:

(1) There are binding sites located on the surface of the adsorbent that are specific for the adsorbate molecule

(2) There are no interactions between the adsorbate molecules bound to the adsorbent surface

(3) The adsorption energy does not depend on the surface covered by adsorbate molecules 
TABLE 1: Estimated values of the parameters of the TOU model and goodness-of-fit measures of adsorption kinetics for DR80 and DB1 removal using Pec-Ca for initial dye concentrations $C_{0}$ of $5,10,15$, and $20 \mathrm{mg} / \mathrm{L}$.

\begin{tabular}{lcccccccc}
\hline & \multicolumn{3}{c}{ DR80 } & \multicolumn{3}{c}{ DB1 } \\
\hline$C_{0}(\mathrm{mg} / \mathrm{L})$ & 5 & 10 & 15 & 20 & 5 & 10 & 15 & 20 \\
$q_{e}(\mathrm{mg} / \mathrm{g})$ & 2.0609 & 4.4638 & 6.7431 & 9.2135 & 2.2799 & 4.7308 & 7.2001 & 9.7113 \\
$n$ & 1.1577 & 1.48 & 1.1369 & 1.4678 & 2.1685 & 1.2683 & 1.4042 & 2.0232 \\
$k_{n}\left((\mathrm{mg} / \mathrm{g})^{1-n} / \mathrm{min}\right)$ & 0.0792 & 0.0508 & 0.135 & 0.0847 & 0.2855 & 0.1474 & 0.0824 & 0.0517 \\
$a$ & 0.014 & 0.05 & 0.024 & 0.112 & 0.874 & 0.06 & 0.074 & 0.542 \\
$\lambda(1 / \mathrm{min})$ & 0.0888 & 0.1042 & 0.1753 & 0.2394 & 0.7479 & 0.2236 & 0.1831 & 0.5297 \\
$\sigma(\mathrm{mg} / \mathrm{g})$ & 0.0276 & 0.0406 & 0.0335 & 0.0865 & 0.0943 & 0.0718 & 0.11 & 0.2141 \\
$\tau(\mathrm{mg} / \mathrm{g})-1$ & 0.0654 & 0.0889 & 0.0566 & 0.125 & 0.0771 & 0.1074 & 0.1817 & 0.208 \\
Half-life $t_{1 / 2}(\mathrm{~min})$ & 8.2496 & 7.8957 & 4.1484 & 3.4195 & 1.4277 & 3.4062 & 4.3694 & 1.9048 \\
Median $(\mathrm{min})$ & 59.5222 & 138.3391 & 42.9594 & 71.3158 & 93.7445 & 34.4779 & 56.5945 & 139.0425 \\
Log-likelihood & 5.9069 & 5.8103 & 6.0401 & 5.0367 & 6.6539 & 5.0189 & 4.2146 & 4.5655 \\
$R^{2}$ & 0.9865 & 0.9952 & 0.9983 & 0.9982 & 0.9868 & 0.9884 & 0.9855 & 0.9857 \\
MSE (mg/g) & 0.0233 & 0.0371 & 0.0266 & 0.0537 & 0.0233 & 0.0909 & 0.2686 & 0.4656 \\
$\Delta q(\%)$ & 2.8672 & 1.8046 & 0.8658 & 0.9313 & 2.5519 & 2.6365 & 3.3137 \\
\hline
\end{tabular}

TABLE 2: Kinetic parameters and coefficients of determination of the PFO, PSO, and PNO models for the adsorption of DR80 and DB1 by Pec-Ca for initial dye concentrations $C_{0}$ of $5,10,15$, and $20 \mathrm{mg} / \mathrm{L}$.

\begin{tabular}{|c|c|c|c|c|c|c|c|c|c|}
\hline Model & $C_{0}(\mathrm{mg} / \mathrm{L})$ & $n$ & $q_{e}(\mathrm{mg} / \mathrm{g})$ & $\begin{array}{l}\text { DR80 } \\
k_{n}\left((\mathrm{mg} / \mathrm{g})^{1-n} / \mathrm{min}\right)\end{array}$ & $R^{2}$ & $n$ & $q_{e}(\mathrm{mg} / \mathrm{g})$ & $\begin{array}{l}\text { DB1 } \\
k_{n}\left((\mathrm{mg} / \mathrm{g})^{1-n} / \mathrm{min}\right)\end{array}$ & $R^{2}$ \\
\hline $\mathrm{PFO}$ & & 1 & 2.0595 & 0.0765 & 0.9859 & 1 & 2.2323 & 0.1982 & 0.9816 \\
\hline PSO & 5 & 2 & 2.1476 & 0.0735 & 0.9709 & 2 & 2.2681 & 0.2724 & 0.9867 \\
\hline PNO & & 1.1527 & 2.0669 & 0.0765 & 0.9868 & 2.1977 & 2.2791 & 0.3002 & 0.9868 \\
\hline $\mathrm{PFO}$ & & 1 & 4.3856 & 0.0755 & 0.988 & 1 & 4.7149 & 0.1763 & 0.9879 \\
\hline PSO & 10 & 2 & 4.5888 & 0.0325 & 0.9893 & 2 & 4.7885 & 0.1202 & 0.9858 \\
\hline PNO & & 1.4318 & 4.4495 & 0.0533 & 0.9953 & 1.3035 & 4.7278 & 0.1490 & 0.9885 \\
\hline $\mathrm{PFO}$ & & 1 & 6.7332 & 0.1549 & 0.9981 & 1 & 7.1365 & 0.135 & 0.9825 \\
\hline PSO & 15 & 2 & 6.8559 & 0.0683 & 0.9923 & 2 & 7.3142 & 0.0468 & 0.9827 \\
\hline $\mathrm{PNO}$ & & 1.1310 & 6.7407 & 0.136 & 0.9983 & 1.4527 & 7.1934 & 0.0809 & 0.9857 \\
\hline $\mathrm{PFO}$ & & 1 & 9.1636 & 0.1523 & 0.9951 & 1 & 9.5404 & 0.178 & 0.9765 \\
\hline PSO & 20 & 2 & 9.3466 & 0.0466 & 0.9952 & 2 & 9.7267 & 0.051 & 0.9857 \\
\hline PNO & & 1.4260 & 9.2168 & 0.0878 & 0.9982 & 2.0031 & 9.7275 & 0.0509 & 0.9857 \\
\hline
\end{tabular}

(4) The maximum adsorption is limited to a monolayer of adsorbate molecules bound to the adsorbent surface

(5) The concentration of the adsorbate is constant

The Pec-Ca-DR80 system does not satisfy the above assumptions. For example, [37] reported that the attachment of the first dye molecule to the adsorbent promoted the formation of aggregates on the adsorbent surface. This is attributed to aromatic rings and other functional groups of the dye molecules, which generate molecular planarity and lead to the formation of $\mathrm{H}$-type aggregates $[38,39]$. Consequently, the DR80 dye molecules can attach to the adsorbent in the form of monomers, dimers, multimers, and so on. Additionally, pectin contains smooth and hairy regions that have different molecular-level hydrophobicity and affinity for DR80. This explains the irregularity in the values of $n$ and $k_{n}$ shown in Table 1.

On the other hand, consider a fractional maximum likelihood estimation (MLE) of the order $n$ (see Table 1). Figure 5 shows the likelihood function and coefficient of determination relative to $n$ for the initial DR80 dye concentration of $20 \mathrm{mg} / \mathrm{L}$. Here, $n=1.47$ is the MLE for the TOU model. This optimal value results in a high coefficient of determination $\left(R^{2}=0.998\right.$; see Figure 5$)$. The orders $n=1$ and $n=2$ have lower likelihoods and coefficients of determination. Therefore, the PFO and PSO models are discarded.

On the other hand, Table 1 shows that the $\lambda$ values increase as the initial dye concentration increases, suggesting that high dye removal values will result in a larger product 


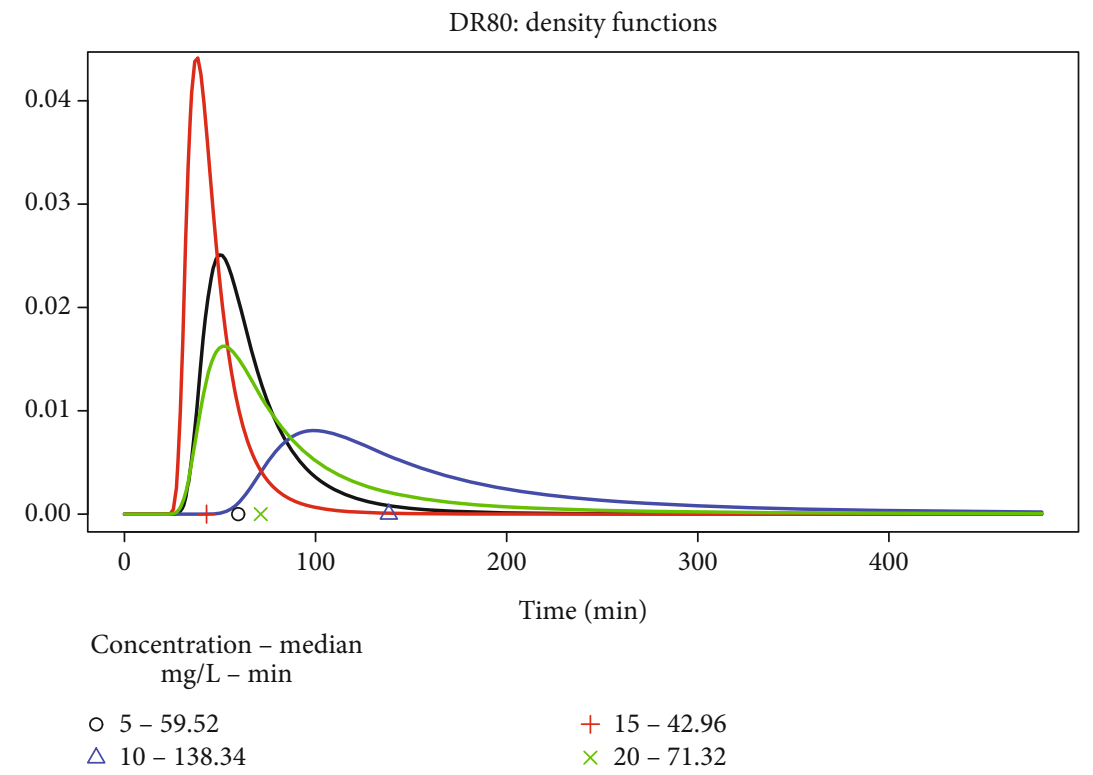

DB1: density functions

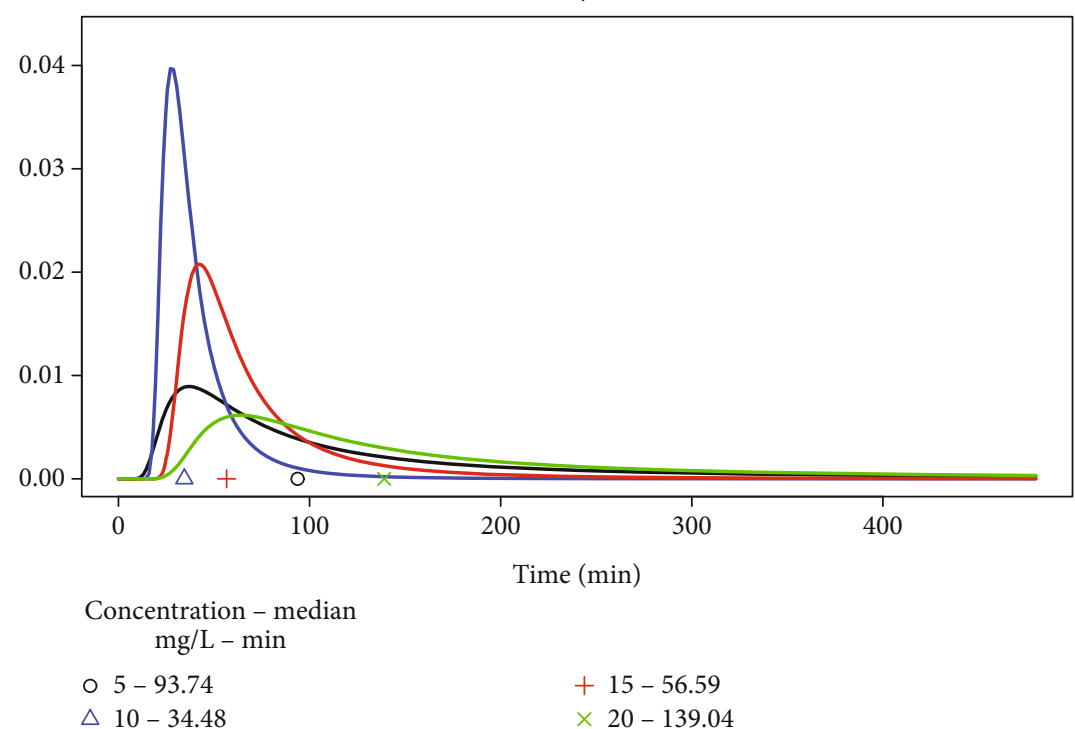

FIGURE 4: Density functions of the time $\left(t_{e}\right)$ needed to reach the $q_{e}$ value at equilibrium for DR80 (top) and DB1 (bottom) at different initial concentrations $(5,10,15$, and $20 \mathrm{mg} / \mathrm{L})$.

$\lambda q_{e}$. This is because a greater difference in the DR80 concentration between the aqueous phase and the adsorbent will result in a greater driving force (chemical potential); thus, the initial adsorption rate will be faster for the initial concentration of $20 \mathrm{mg} / \mathrm{L}$ than for the concentration of $5 \mathrm{mg} / \mathrm{L}$. In addition, the $\mathrm{pH}$ value at which the adsorption kinetics were measured $(\mathrm{pH}=3)$ significantly affected the dye removal efficiency. At this $\mathrm{pH}, \mathrm{Pec}-\mathrm{Ca}$ retains part of its molecular structure, including a polymeric network in which the galacturonate residues were found primarily in the smooth region of pectin chelate calcium ion. Pectin "dimers" are then formed and polymerize, changing the rheological properties of pectin, which can be observed at the macroscopic level in Pec-Ca gel $[40,41]$. Since the $\mathrm{pKa}$ of pectin is between 2.8 and 4.1 [42], at $\mathrm{pH}=3$, some Pec-Ca molecules have a sufficient positive charge to attract DR80 molecules, whose charge is negative at $\mathrm{pH} 3$ due to the presence of six sulfonate groups (see Figure 2). Additionally, the presence of hydrogen bonds between the functional groups of the dye and its tautomers (sulfonate, amide, azo, and hydrazone) and the functional groups of pectin (carboxyl, hydroxyl, and oxygen atoms of glycosidic and hemiacetal bonds) cause DR80 to have a high affinity for pectin. Furthermore, Yoshida's interactions between the electrons of the aromatic rings of the dye and the hydrogen atoms of the sugar residues contained in the polysaccharide reinforce the binding of DR80 to Pec-Ca [43].

Figure 6 shows the variation in $q_{t}$ as a function of time for DB1 and the theoretical kinetics obtained with the TOU model. The theoretical curve adequately reproduces the experimental data, and the corresponding parameters are included in Table 1. 

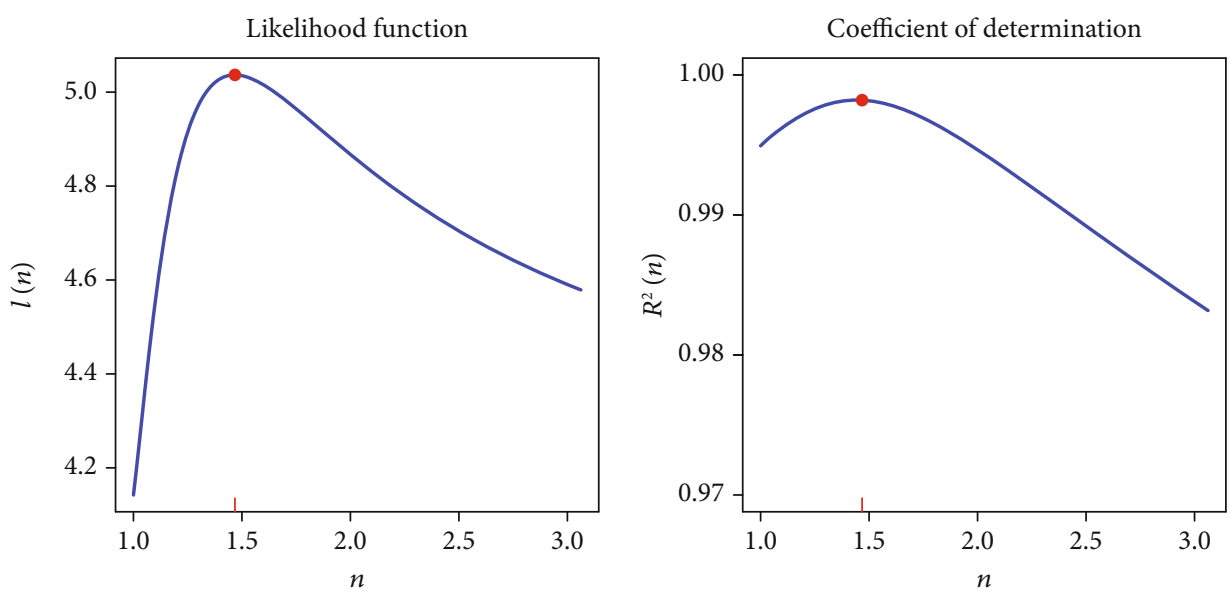

MLE

- $n=1.47$

FIGURE 5: Indicators of the fit of the TOU model for DR80 adsorption (initial concentration $=20 \mathrm{mg} / \mathrm{L}$ ) at different values of $n$ : log-likelihood (left) and coefficient of determination (right).

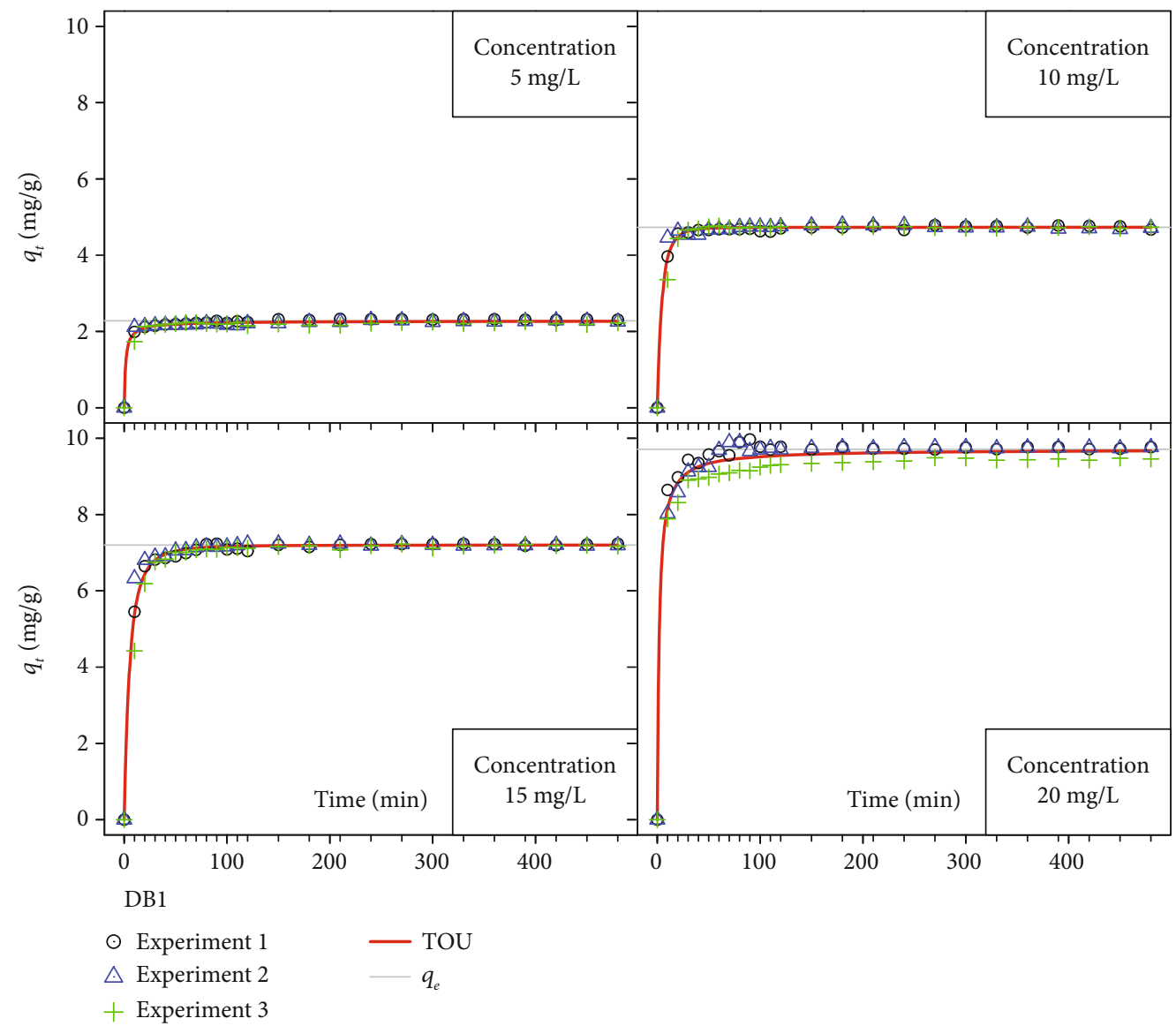

FIGURE 6: Experimental adsorption kinetics for the removal of DB1 by Pec-Ca at different initial dye concentrations and the theoretical curves generated by applying the TOU model to the experimental data.

Table 1 shows that the MSE values in the Pec-Ca-DB1 system are lower than those in the Pec-Ca-DR80 system. Meanwhile, the $R^{2}$ values of the Pec-Ca-DB1 system are slightly lower than those of the Pec-Ca-DR80 system, while the $\Delta q$ values are larger. However, the obtained values are acceptable from a statistical point of view and confirm that the TOU model adequately describes the experimental data. 
As the DB1 concentration increases, the values of $q_{e}$ also increase. Interestingly, the $q_{e}$ values obtained for DB1 are higher than those found in the Pec-Ca-DR80 system. It is important to mention that the adsorption kinetics of DB1 were measured at $\mathrm{pH} 2$, resulting in the protonation of PecCa because the carboxyl groups of the galacturonate residues contained primarily in the smooth region of this biopolymer are protonated, and a considerable portion of Pec-Ca is transformed into pectinic acid. This protonation favors the formation of polygalacturonic acid regions, which repulse DB1 molecules to a lesser extent than the calcium galacturonate contained in Pec-Ca. This favors the formation of hydrogen bonds between the functional groups of pectin (as mentioned previously) and the sulfonate, amide, methoxy, and keto groups of DB1. Moreover, Yoshida-type interactions similar to those in the Pec-Ca-DR80 system increase the affinity of DB1 for the polysaccharide. In contrast to the Pec-Ca-DR80 system, the Pec-Ca-DB1 system has higher pseudoorder values closer to $n=2$, suggesting that the main type of binding in the Pec-Ca-DB1 system is chemisorption. Consequently, DB1 shows greater affinity for Pec-Ca than DR80, consistent with the results discussed above.

The $k_{n}$ values reported in Table 1 decrease as the DB1 concentration increases. Reference [44] found that pectin and alginic acid form a gel in a similar manner with the calcium ion. In the same way, [31] found that when DB1 is removed from an aqueous medium containing alginic acid, DB1 tends to form aggregates on the surface of alginic acid. Considering the structural similarity of these polysaccharides, molecular aggregates of DB1 are expected to form on the surface of pectin as the concentration of DB1 increases. As a consequence, the diffusion rate of DB1 molecules between the liquid and the pectin molecular surface will be decreased.

In the same way, the $\lambda$ values in the Pec-Ca-DB1 system are greater than those in the Pec-Ca-DR80 system, suggesting less repulsion between DB1 and pectinic acid than between DR80 and pectinic acid. The forces involved in DB1 adsorption by Pec-Ca (electrostatic interaction, hydrogen bonding, and hydrophobic interactions between the dye and the neutral sugars in the hairy regions of pectin) are stronger than those involved in the Pec-Ca-DR80 system. Furthermore, Figure 2 shows that the DB1 molecules are smaller than the DR80 molecules, which allows them to penetrate more deeply into the spaces between the hairy and smooth regions of pectin [45]. These factors result in higher initial adsorption rates in the Pec-Ca-DB1 system compared to the Pec-Ca-DB80 system. Thus, as a general trend, the time required to achieve half of $q_{e}\left(t_{1 / 2}\right)$ will be shorter in the Pec-Ca-DB1 system than in the Pec-Ca-DR80 system (see Table 1).

It is important to note that in all cases, these times are small $\left(t_{1 / 2}\right.$ values between 3.41 and $8.25 \mathrm{~min}$ in the Pec-CaDR80 system and between 1.42 and $4.36 \mathrm{~min}$ in the Pec-CaDB1 system). Thus, if Pec-Ca is used to remove dyes in batch mode, the dye concentration can be reduced by half in these short time periods. That is, Pec-Ca can be used to rapidly remove a considerable amount of colorant in a short period of time, which is often the main objective when dealing with accidents of environmental concern involving highly polluting compounds like the dyes used in this study.

As shown in Table 1, the variation in $q_{t}$ with respect to time (as estimated by the $\sigma$ parameter) is higher in the Pec-Ca-DB1 system than in the Pec-Ca-DR80 system. Furthermore, the maximum variability of the adsorption process $(\tau)$ obtained using the TOU model is larger in the Pec-Ca-DB1 system than in the Pec-Ca-DR80 system.

4.2. Fluoride Removal by Xant-Al. The fluoride adsorption kinetics at different initial concentrations $(1-5 \mathrm{mg} / \mathrm{L})$ are depicted in Figure 7, and the estimated parameter values obtained from the application of the TOU model to the experimental kinetics are shown in Table 3. As shown in Figure 7 , the $q_{t}$ value increases during the first $20-30 \mathrm{~min}$, and the Xant-Al-fluoride system reaches equilibrium after approximately $40 \mathrm{~min}$. The $R^{2}$, MSE, and $\Delta q$ values indicate that the fit of the theoretical curve to the experimental data is acceptable.

The PFO, PSO, and PNO models were also fit to the fluoride adsorption data, and their estimated parameters are shown in Table 4 . The reported $R^{2}$ values for these models are similar to those obtained using the TOU model (Table 3). Although the goodness of fit is similar for the TOU, PFO, PSO, and PNO models, the estimated parameters of the TOU model were obtained by the maximum likelihood method, whereas those of the PFO, PSO, and PNO models were obtained by maximizing the coefficient of determination $R^{2}$. It is worth noting that a similar goodness of fit does not necessarily imply similar estimated parameters (see the values of the parameter $k_{n}$ for the initial fluoride concentration of $5 \mathrm{mg} / \mathrm{L}$ in Tables 3 and 4). The parameter estimates for $k_{n}$ obtained using the PSO and PNO models are sensitive and tend toward high numerical values. Although the values of $R^{2}$ are close to one, the kinetic parameters for the PSO and PNO models are more difficult to interpret from a physical context than those obtained using the TOU model. Although a high coefficient of determination is desirable, it should not be used as a criterion to rank the quality of a particular model. Moreover, the proposed methodology is numerical and computationally stable.

As for the previously discussed systems, the value of $q_{e}$ increases as the initial concentration of fluoride increases, resulting in $q_{e}$ values ranging from 0.417 to $1.911 \mathrm{mg} / \mathrm{g}$ at initial fluoride concentrations in the range of $1-5 \mathrm{mg} / \mathrm{L}$.

Reference [20] reported that Xant-Al is formed by the reaction of xanthan with $\mathrm{AlCl}_{3}$ in aqueous media. This product is a gel that, when lyophilized, produces a powder with a strong ability to remove different anions. Fluorides are negatively charged and consequently can be removed from aqueous environments by Xant-Al. This adsorbent contains the polyhydroxyoxoaluminum clusters $\left[\mathrm{Al}_{13} \mathrm{O}_{4}(\mathrm{OH})_{24}\right.$ $\left.\left(\mathrm{H}_{2} \mathrm{O}\right)_{12}\right] \quad\left[\mathrm{SO}_{4}\right]_{4} \cdot 19 \mathrm{H}_{2} \mathrm{O}$ (denominated as CAL-13) and $\left[\mathrm{Al}_{30} \mathrm{O}_{8}(\mathrm{OH})_{56}\left(\mathrm{H}_{2} \mathrm{O}\right)_{26}\right] \quad\left[\mathrm{SO}_{4}\right]_{9} x \mathrm{H}_{2} \mathrm{O}$ (denominated as CAL-30) [46, 47]. These clusters act as linkers between xanthan molecules to form a polymeric network that possesses a net positive charge that is variable and depends on the proportions of CAL-13 and CAL-30 in Xant-Al. 


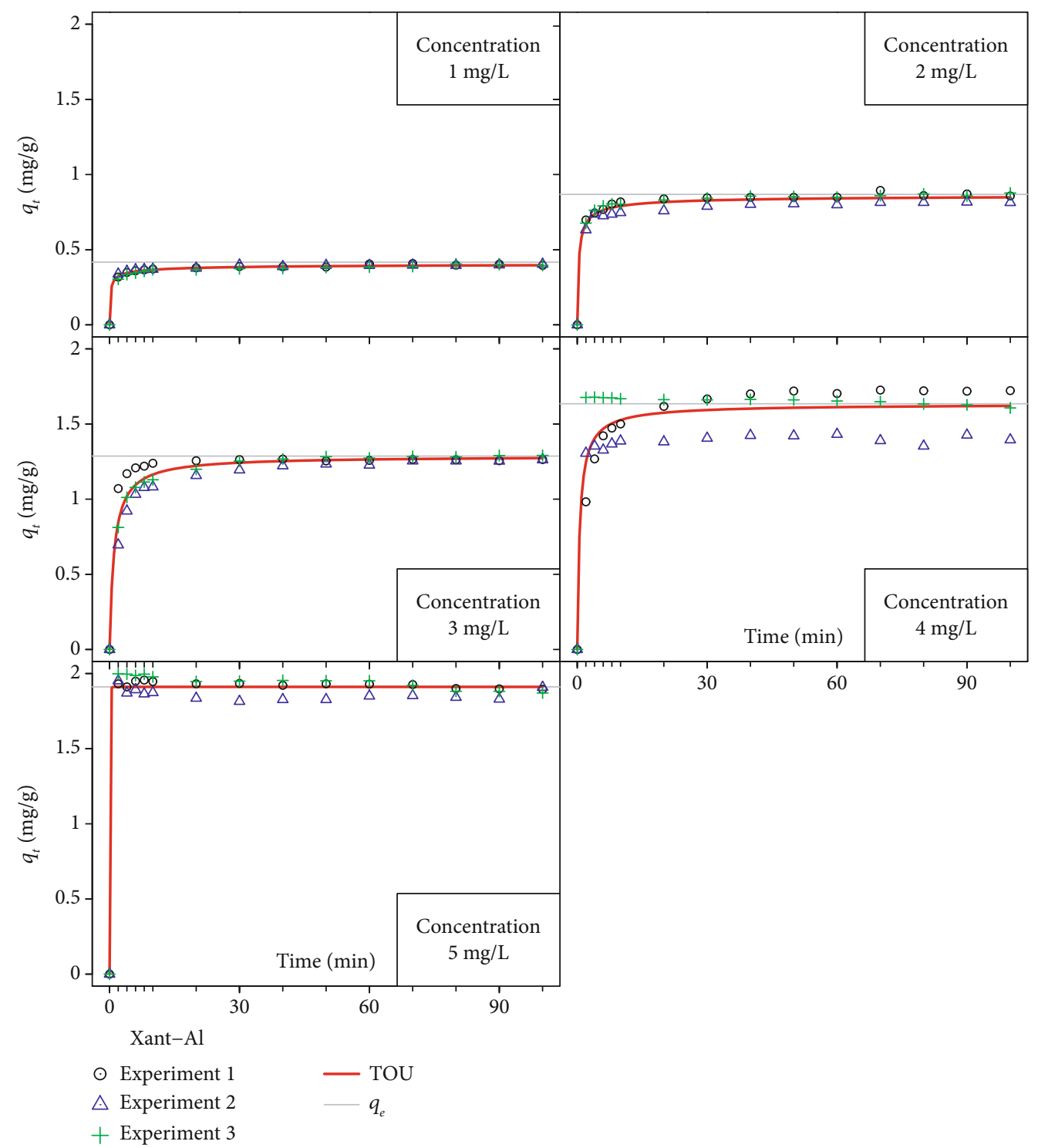

FIgURE 7: Kinetics of fluoride adsorption by Xant-Al for five initial fluoride concentrations and the theoretical curve obtained from the TOU model.

Consequently, negatively charged fluorides have a strong affinity for Xant-Al.

Interestingly, at high fluoride concentrations, the variability in kinetics increases notably. The factors that cause this variability can be extrinsic (inherent to the technique used to quantify fluoride in the aqueous environment) or intrinsic (dependent on physicochemical factors such as $\mathrm{pH}$, ionic strength, and the presence of metal ions that can act as Lewis acids and favor the formation of coordination compounds). Regarding extrinsic factors, all materials used for quantification should be made of inert plastic; the use of glass would cause a decrease in the fluoride concentration in aqueous solution due to the presence of silicon dioxide, resulting in the overestimation of the $q_{e}$ values.

Additionally, the standard curve used to quantify fluoride based on the interpolation of the potentials measured by the ion-selective electrode is not linear; thus, a logarithmic transformation must be applied to linearize the stan- dard curve, which adds uncertainty to the quantification of fluoride.

Reference [21] reported that the presence of $\mathrm{Al}(\mathrm{III})$ at concentrations greater than $3 \mathrm{mg} / \mathrm{L}$ negatively affects the quantification of fluoride in water. Although Xant-Al was washed five times in this study to remove excess $\mathrm{Al}$ ion, it can be dissolved in an aqueous medium at this concentration level and affect the measurement, especially at high fluoride concentrations. Regarding intrinsic factors, the dissociation of nearby water molecules by the $\mathrm{Al}$ atoms in the CAL-13 and CAL-30 clusters (they act as Arrhenius acids) generates a local $\mathrm{pH}$ lower than that of bulk solution. It is important to mention that the initial $\mathrm{pH}$ was adjusted to 7.0 ; after the addition of Xant-Al, the $\mathrm{pH}$ decreased and reached a final value of 3.0 at the end of the kinetic experiment. Thus, the variation in the buffering capacity of the added buffer during the determination of fluoride concentration caused significant randomness in the obtained values of $q_{e}$. 
TABLE 3: Estimated parameter values of the TOU model and goodness-of-fit measures for the adsorption kinetics of fluoride by Xant-Al at initial fluoride concentrations of $1,2,3,4$, and $5 \mathrm{mg} / \mathrm{L}$.

\begin{tabular}{|c|c|c|c|c|c|}
\hline & & & Xant-Al & & \\
\hline$C_{0}(\mathrm{mg} / \mathrm{L})$ & 1 & 2 & 3 & 4 & 5 \\
\hline$q_{e}(\mathrm{mg} / \mathrm{g})$ & 0.4169 & 0.8679 & 1.2866 & 1.6348 & 1.9111 \\
\hline$n$ & 3.5304 & 2.6629 & 2.0054 & 2.1147 & 1 \\
\hline$k_{n}\left((\mathrm{mg} / \mathrm{g})^{1-n} / \mathrm{min}\right)$ & 75.7363 & 4.1397 & 0.7427 & 1.0171 & 12.01 \\
\hline$a$ & 20.942 & 5.439 & 0.962 & 1.961 & 0 \\
\hline$\lambda(1 / \mathrm{min})$ & 8.2763 & 3.2708 & 0.9569 & 1.7592 & 12.01 \\
\hline$\sigma(\mathrm{mg} / \mathrm{g})$ & 0.0696 & 0.0803 & 0.0667 & 0.1929 & 0.246 \\
\hline$\tau(\mathrm{mg} / \mathrm{g})^{-1}$ & 0.0171 & 0.0314 & 0.0482 & 0.1028 & 0.0502 \\
\hline Half-life $t_{1 / 2}(\mathrm{~min})$ & 0.2281 & 0.3983 & 1.0472 & 0.5944 & 0.0577 \\
\hline Median (min) & 418.3113 & 88.118 & 40.8972 & 16.7791 & 0.3358 \\
\hline Log-likelihood & 9.7554 & 8.2108 & 7.025 & 5.5563 & 5.9835 \\
\hline$R^{2}$ & 0.9936 & 0.9841 & 0.9648 & 0.8728 & 0.9898 \\
\hline $\operatorname{MSE}(\mathrm{mg} / \mathrm{g})^{2}$ & 0.0005 & 0.0062 & 0.0326 & 0.1986 & 0.0212 \\
\hline$\Delta q(\%)$ & 2.2515 & 3.5125 & 6.2594 & 10.7724 & 2.6762 \\
\hline
\end{tabular}

TABLE 4: Kinetic parameters and coefficients of determination for the PFO, PSO, and PNO models of fluoride adsorption by Xant-Al for initial fluoride concentrations of $1,2,3,4$, and $5 \mathrm{mg} / \mathrm{L}$.

\begin{tabular}{|c|c|c|c|c|c|}
\hline$C_{0}(\mathrm{mg} / \mathrm{L})$ & model & $n$ & $q_{e}(\mathrm{mg} / \mathrm{g})$ & $k_{n}\left((\mathrm{mg} / \mathrm{g})^{1-\mathrm{n}} / \mathrm{min}\right)$ & $R^{2}$ \\
\hline \multirow{3}{*}{1} & $\mathrm{PFO}$ & 1 & 0.3837 & 0.8082 & 0.9734 \\
\hline & PSO & 2 & 0.3946 & 4.7417 & 0.9908 \\
\hline & PNO & 3.7317 & 0.423 & 94.3324 & 0.9938 \\
\hline \multirow{3}{*}{2} & $\mathrm{PFO}$ & 1 & 0.8225 & 0.764 & 0.9678 \\
\hline & PSO & 2 & 0.8461 & 2.0937 & 0.983 \\
\hline & PNO & 2.7063 & 0.8696 & 4.3741 & 0.9841 \\
\hline \multirow{3}{*}{3} & $\mathrm{PFO}$ & 1 & 1.2313 & 0.5094 & 0.9464 \\
\hline & PSO & 2 & 1.2785 & 0.8019 & 0.9652 \\
\hline & PNO & 2.111 & 1.2857 & 0.8386 & 0.9653 \\
\hline \multirow{3}{*}{4} & $\mathrm{PFO}$ & 1 & 1.5591 & 0.871 & 0.8687 \\
\hline & PSO & 2 & 1.5951 & 1.4379 & 0.8775 \\
\hline & PNO & 2.2156 & 1.6053 & 1.6465 & 0.8776 \\
\hline \multirow{3}{*}{5} & $\mathrm{PFO}$ & 1 & 1.9111 & 32.4142 & 0.9897 \\
\hline & PSO & 2 & 1.9111 & 81847.546 & 0.9897 \\
\hline & $\mathrm{PNO}$ & 1.0359 & 1.911 & 313.5464 & 0.9897 \\
\hline
\end{tabular}

As shown in Figure 7 and Table 3, the combined extrinsic and intrinsic factors give rise to variability in the estimated values of $q_{e}, k_{n}$, and $n$. In particular, the estimates of $n$ are high (close to 2), suggesting chemisorption between Xant$\mathrm{Al}$ and fluoride due to the strong electrostatic interaction between these two ions (see Table 3). However, [48] reported that $n$ values greater than 2 result from an increase in the number of adsorbate (tetracycline) binding sites due to the breakdown of intra- and intermolecular interactions in chitosan (adsorbent) as a result of the protonation of different regions of this polymer dissolved in aqueous solution.

In contrast to the findings observed for chitosan, in the Xant-Al-fluoride system, an opposite trend is observed as the concentration of fluoride increases. This indicates that Xant-Al has a defined number of binding sites that are directly related to the amounts of CAL-13 and CAL-30 clusters present in Xant-Al. When low concentrations of fluoride are added to Xant-Al, the adsorption is governed by ionic bonds between the fluoride ions and positively charged clusters, resulting in high $n$ valuess. When the fluoride concentration increases, the finite binding sites on the CAL-13 and CAL-30 clusters become occupied, and the ability of the fluoride anions remaining in bulk solution to bind to the surface of Xant-Al is reduced. In addition, the initial experimental $\mathrm{pH}$ (7.0) was lower than the point of zero charge of Xant$\mathrm{Al}(\mathrm{pzc}=10.2)$, suggesting that this material had a positive 


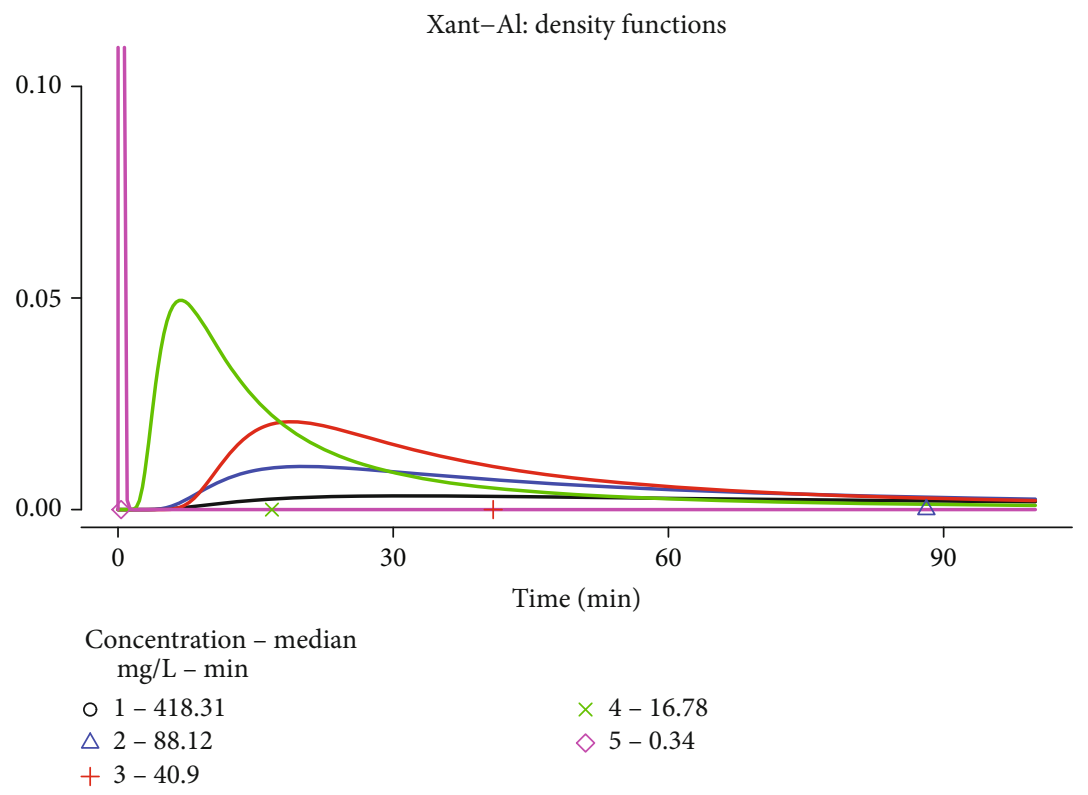

Figure 8: Density functions of the time $\left(t_{e}\right)$ needed to reach $q_{e}$ at equilibrium for the adsorption of fluoride with Xant-Al in an aqueous medium for five different initial fluoride concentrations.

charge at the start of each adsorption experiment. The experimentally measured $\mathrm{pH}$ values at the end of the kinetic experiments were all around 3.0; this $\mathrm{pH}$ would have promoted the ionization of Xant-Al, diminishing the net charge on the Xant-Al molecular surface. These results suggest that $n$ decreases (from 3.5 to 1.0 ) as the fluoride concentration increases (see Table 3). In this sense, it is the Coulombic attraction between Xant- $\mathrm{Al}$ and fluoride that gives rise to the small $t_{1 / 2}$ values (0.058-1.047 min) since binding between oppositely charged species occurs quickly.

Figure 8 shows the density functions of the time $\left(t_{e}\right)$ needed to reach $q_{e}$ at equilibrium for five fluoride concentration levels. This figure shows that the initial fluoride concentration influences the shape of the density function. The equilibrium time, the dispersion of the density function, and the median all decrease considerably with increasing fluoride concentration. This can be attributed to the electrostatic nature of the adsorption process, which releases energy and takes place in a very short time. A greater amount of "reagent" such as fluoride ion is expected to produce a greater release of energy in a relatively short time to reach the equilibrium state.

4.3. Cadmium Removal by Reed Leaves. The third system to which the TOU model was applied is cadmium adsorption by reed leaves, as reported by [22]. This group used several plant materials, including reed leaves, to eliminate $\mathrm{Cd}(\mathrm{II})$ at initial concentrations of $1,2,4$, and $6 \mathrm{mg} / \mathrm{L}$ in aqueous media. Figure 9 shows the experimental adsorption kinetics and the theoretical curve resulting from the application of the TOU model to these data.

Reference [49] reported that reed leaf contains a considerable percentage of lignin (8.74\%), which has been shown to form chelates with different divalent ions such as $\mathrm{Cd}(\mathrm{II})$, $\mathrm{Pb}(\mathrm{II}), \mathrm{Cu}(\mathrm{II}), \mathrm{Zn}(\mathrm{II})$, and $\mathrm{Ni}(\mathrm{II})$ [50]. This is due to the pres- ence of functional groups (e.g., hydroxyl and carboxyl groups) in the structures of compounds attached to lignin (e.g., p-coumaryl, coniferyl, and sinapyl alcohol and some organic acids such as ferulic, $p$-coumaric, and $p$-hydroxybenzoic acid) [50, 51]. However, the high contents of cellulose (49.4\%) and hemicellulose (31.5\%), which have little affinity for divalent metal ions, limit the ability of reed leaf to remove cadmium ion from aqueous solution (the $q_{e}$ values obtained range from 0.0476 to $0.2994 \mathrm{mg} / \mathrm{g}$ ). Moreover, the values of the constants obtained are small; consequently, a long time is needed to reach equilibrium compared to the other systems discussed in this work.

As shown in Table 5, the coefficients of determination, MSE, and $\Delta q$ values are acceptable; however, they show a certain level of variability because the lignin, cellulose, and hemicellulose contents vary from one reed leaf to another. Consequently, the number of sites for $\mathrm{Cd}(\mathrm{II})$ adsorption differs for the various $\mathrm{Cd}(\mathrm{II})$ concentration levels considered in this study. Additionally, the $n$ value was found to increase as the concentration of $\mathrm{Cd}(\mathrm{II})$ increases. This trend is similar to that found by [48], which used chitosan to remove tetracycline in an aqueous medium. This behavior was attributed to the protonation of the amino groups of the polysaccharide, resulting in an increase in the number of adsorbate binding sites. Reed leaves can be hydrated to facilitate the access of ionic species present in aqueous solution, including $\mathrm{Cd}(\mathrm{II})$, to the aforementioned functional groups (carboxyl and hydroxyl groups); this phenomenon can explain the magnitude of $n$ values observed in Table 5 .

The PFO, PSO, and PNO kinetic models were fit to the cadmium removal data, and the resulting parameter estimates are shown in Table 6 . The $R^{2}$ value for the PNO model is similar to that obtained using the TOU model (Table 5). Although the goodness of fit is similar for the TOU and PNO models, the kinetic parameters $q_{e}$ and $k_{n}$ of the PNO 


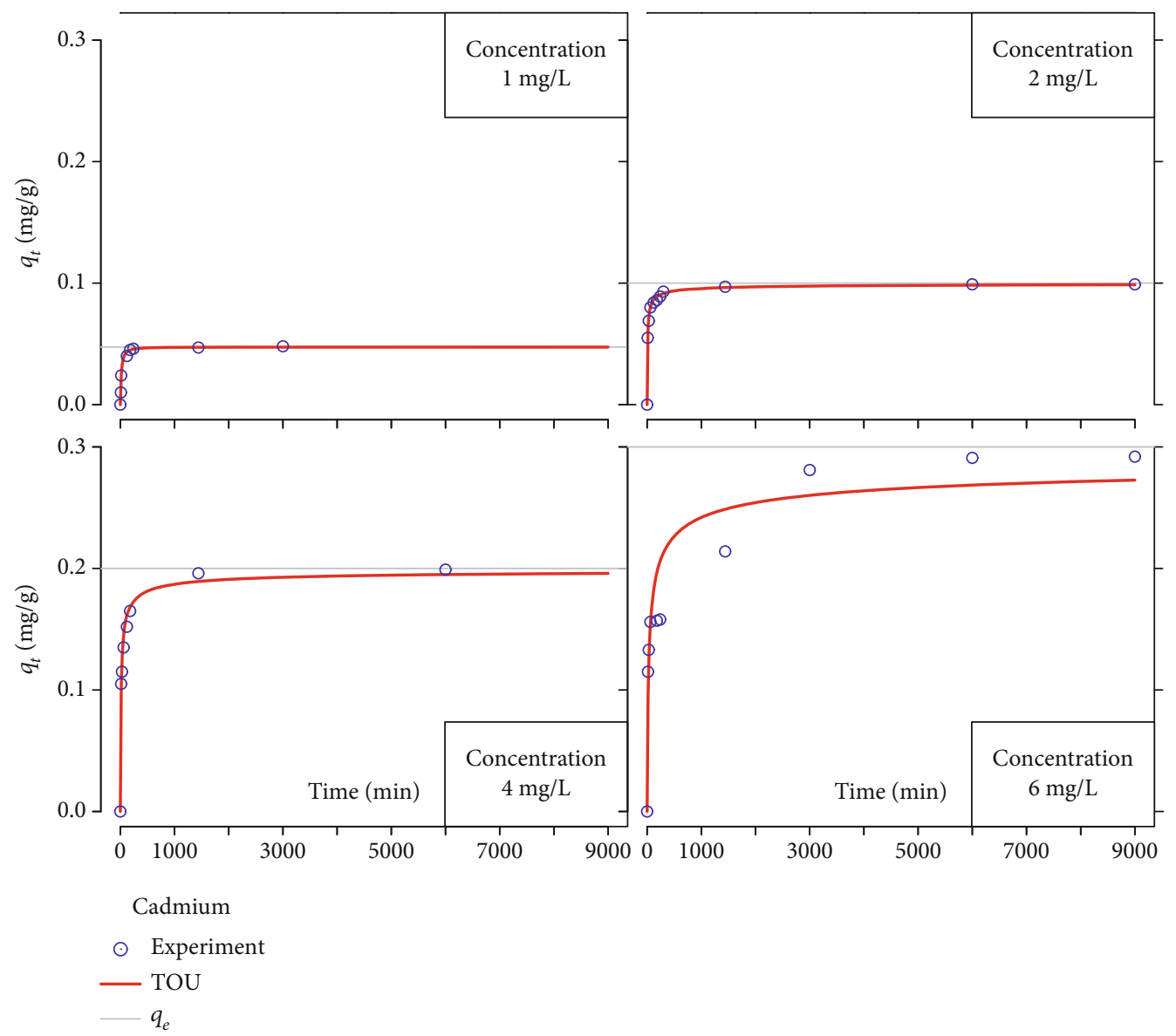

FIGURE 9: Adsorption kinetics for the removal of cadmium by reed leaves for four initial Cd(II) concentrations and the theoretical curve obtained from the TOU model.

TABLE 5: Estimated values of the parameters of the TOU model and goodness-of-fit measures for the adsorption of Cd(II) by reed leaves for initial Cd(II) concentrations of $1,2,4$, and $6 \mathrm{mg} / \mathrm{L}$.

\begin{tabular}{|c|c|c|c|c|}
\hline \multirow[b]{2}{*}{$C_{0}(\mathrm{mg} / \mathrm{L})$} & \multicolumn{4}{|c|}{ Cadmium removal } \\
\hline & 1 & 2 & 4 & 6 \\
\hline$q_{e}(\mathrm{mg} / \mathrm{g})$ & 0.0476 & 0.0995 & 0.1995 & 0.2994 \\
\hline$n$ & 1.8456 & 2.7386 & 2.8269 & 3.8792 \\
\hline$k_{n}\left((\mathrm{mg} / \mathrm{g})^{1-n} / \mathrm{min}\right)$ & 0.7568 & 8.8618 & 1.6844 & 1.2885 \\
\hline$a$ & 0.0488 & 0.2788 & 0.1618 & 0.1152 \\
\hline$\lambda(1 / \mathrm{min})$ & 0.0577 & 0.1604 & 0.0886 & 0.04 \\
\hline$\sigma(\mathrm{mg} / \mathrm{g})$ & 0.0021 & 0.0016 & 0.0037 & 0.0104 \\
\hline$\tau(\mathrm{mg} / \mathrm{g})^{-1}$ & 0.0063 & 0.0028 & 0.0087 & 0.0368 \\
\hline Half-life $t_{1 / 2}(\mathrm{~min})$ & 16.3429 & 8.381 & 15.7408 & 55.2008 \\
\hline Median (min) & 139.23 & 3437.19 & 3890.12 & 11370.4 \\
\hline Log-likelihood & 10.577 & 12.439 & 10.141 & 7.616 \\
\hline$R^{2}$ & 0.9727 & 0.996 & 0.9890 & 0.9051 \\
\hline $\operatorname{MSE}(\mathrm{mg} / \mathrm{g})^{2}$ & $<10^{-4}$ & $<10^{-4}$ & $<10^{-4}$ & $<10^{-4}$ \\
\hline$\Delta q(\%)$ & 28.77 & 2.476 & 5.188 & 18.07 \\
\hline
\end{tabular}

model are sensitive and tend to be higher than those for the TOU model (for instance, compare the estimated parameters for initial $\mathrm{Cd}(\mathrm{II})$ concentrations of 2,4 , and
$6 \mathrm{mg} / \mathrm{L}$ in Tables 5 and 6). Such kinetic parameters are more difficult to interpret from the physicochemical point of view. These findings confirm that the performance of the TOU 
TABLE 6: Kinetic parameters and coefficients of determination for the PFO, PSO, and PNO models for the adsorption of Cd(II) by reed leaves for initial Cd(II) concentrations of $1,2,4$, and $6 \mathrm{mg} / \mathrm{L}$.

\begin{tabular}{|c|c|c|c|c|c|}
\hline$C_{0}(\mathrm{mg} / \mathrm{L})$ & Model & $n$ & $q_{e}(\mathrm{mg} / \mathrm{g})$ & $k_{n}\left((\mathrm{mg} / \mathrm{g})^{1-n} / \mathrm{min}\right)$ & $R^{2}$ \\
\hline \multirow{3}{*}{1} & $\mathrm{PFO}$ & 1 & 0.0465 & 0.036 & 0.9686 \\
\hline & PSO & 2 & 0.0490 & 0.9052 & 0.9814 \\
\hline & $\mathrm{PNO}$ & 1.8245 & 0.0485 & 0.5076 & 0.9819 \\
\hline \multirow{3}{*}{2} & $\mathrm{PFO}$ & 1 & 0.0906 & 0.0687 & 0.9371 \\
\hline & PSO & 2 & 0.095 & 1.1233 & 0.9846 \\
\hline & PNO & 3.1559 & 0.1015 & 26.016 & 0.9981 \\
\hline \multirow{3}{*}{4} & $\mathrm{PFO}$ & 1 & 0.183 & 0.0344 & 0.9001 \\
\hline & PSO & 2 & 0.1925 & 0.2721 & 0.9729 \\
\hline & $\mathrm{PNO}$ & 3.6419 & 0.209 & 6.8439 & 0.9939 \\
\hline \multirow{3}{*}{6} & $\mathrm{PFO}$ & 1 & 0.2318 & 0.0262 & 0.7009 \\
\hline & PSO & 2 & 0.255 & 0.0991 & 0.819 \\
\hline & $\mathrm{PNO}$ & 17.0668 & 0.6597 & 47.0188 & 0.9567 \\
\hline
\end{tabular}

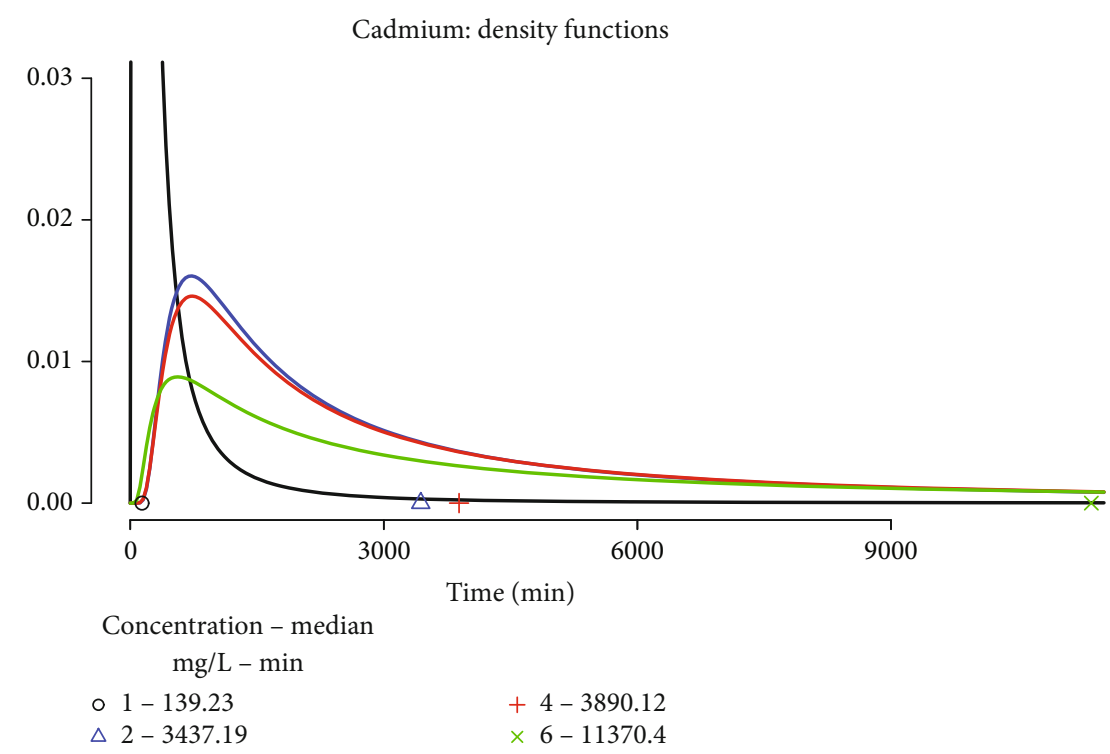

Figure 10: Density functions of the time $\left(t_{e}\right)$ needed to reach equilibrium for the removal of Cd(II) using reed leaves at initial Cd(II) concentrations of $1,2,4$, and $6 \mathrm{mg} / \mathrm{L}$.

model is competitive with other models in terms of its ability to fit experimental data. In addition, the TOU model is numerically and computationally stable.

The $t_{1 / 2}$ values for this system also show apparently irregular behavior; however, the origin of this variability is the same as that discussed for $n$ and can be explained by the compositional heterogeneity of lignocellulosic materials such as reed leaves [49]. Evidently, the average $t_{1 / 2}$ value for this system is greater than that of the Pec-Ca and Xant-Al systems (see Table 5).

Figure 10 shows the $t_{e}$ density functions for the four initial Cd(II) concentrations. As the initial Cd(II) concentration increases, the density functions of the time required to reach equilibrium show greater dispersion. Likewise, the median and the time needed to reach equilibrium increase signifi- cantly with increasing $\mathrm{Cd}(\mathrm{II})$ concentration. For instance, increasing the $\mathrm{Cd}$ (II) concentration from 4 to $6 \mathrm{mg} / \mathrm{L}$ generates a significant increase in the median $t_{e}$ value from 3,890 to $11,370 \mathrm{~min}$. The proposed cadmium removal mechanism implies a global formation constant $\left(K_{F}\right)$ in which organic acids and alcohols chelate $\mathrm{Cd}(\mathrm{II})$. Thus, an increase in one of the reactants $\left(\mathrm{Cd}^{+2}\right)$ can be expected to promote an increase in the respective $\mathrm{Cd}(\mathrm{II})$ complex concentration. However, this process requires a longer time when the added amount of Cd(II) increases.

It is important to note that the natures of both the pollutant and adsorbent are essential in the removal of pollutants such as dyes, fluoride, and $\mathrm{Cd}(\mathrm{II})$ by the materials used in this study (Pec-Ca, Xant-Al, and reed leaves, respectively). Thus, it is necessary to use models that adequately describe the 
observed adsorption kinetics. In this sense, the TOU model, which includes models of different pseudoorders (i.e., the PFO, PSO, and PNO (general) models) is a good option for the studied systems, despite the different chemical characteristics of the adsorbents and pollutants in these systems. The findings suggest that the TOU model can be widely applied for a variety of adsorbate-adsorbent systems in aqueous environments.

\section{Conclusions}

The proposed TOU model based on the transformation of the OU stochastic process consistently shows good performance for describing the adsorption kinetics of different pollutants by distinct materials in aqueous media. The TOU model includes stochastic versions of the PFO, PSO, and PNO models.

The TOU model successfully describes the approach to equilibrium and the variability inherent in experimental adsorption kinetics. The proposed model has wide application potential to the removal of pollutants such as DR80 and DB1 dyes, fluoride, and cadmium ions by Pec-Ca, Xant-Al, and reed leaves, respectively.

By explicitly including an uncertainty component, the proposed model has the advantage of providing the probability distribution of the time needed to reach equilibrium, which reduces cost and facilitates the removal of pollutants in the shortest time possible. Additionally, the estimation procedure incorporated in the model considers all the experimental kinetic replicates. The MLE method does not require knowledge of the $q_{e}$ value. Furthermore, due to the predictive capacity of the model, it is not necessary that kinetic experiments be conducted up until the point that equilibrium is reached.

The proposed model is the first stochastic-based model that can characterize different adsorption kinetic behaviors and estimate the probability distribution of time at which the maximum removal of different pollutants is achieved.

For future investigations, the structure of the proposed model allows its extension to a wide family of adsorption kinetic systems.

\section{Data Availability}

To request data about direct blue 1 , direct red 80 , and fluorides, contact Silvia Rodríguez-Narciso via the email srodrign@correo.uaa.mx. The database on cadmium removal by reed leaves is reported in Sayrafi et al. [22].

\section{Conflicts of Interest}

The authors declared no potential conflicts of interest with respect to the research, authorship, and/or publication of this article.

\section{Acknowledgments}

This work was supported by the Universidad Autónoma de Aguascalientes through grants PIBT19-3, PIM20-5, and PIM20-7.

\section{Supplementary Materials}

The supplemental material for this article is available online (Supplementary Materials). (Supplementary Materials)

\section{References}

[1] Y. Zhou, J. Lu, Y. Zhou, and Y. Liu, "Recent advances for dyes removal using novel adsorbents: a review," Environmental Pollution, vol. 252, pp. 352-365, 2019.

[2] P. Singh and A. Borthakur, "A review on biodegradation and photocatalytic degradation of organic pollutants: a bibliometric and comparative analysis," Journal of Cleaner Production, vol. 196, pp. 1669-1680, 2018.

[3] A. P. Lim and A. Z. Aris, "A review on economically adsorbents on heavy metals removal in water and wastewater," Reviews in Environmental Science and Bio/Technology, vol. 13, pp. 163-181, 2014.

[4] J. O. Tijani, O. O. Fatoba, and L. F. Petrik, "A review of pharmaceuticals and endocrine-disrupting compounds: sources, effects, removal, and detections," Water, Air, \& Soil Pollution, vol. 224, 2013.

[5] J. Simonin and J. Bouté, "Intraparticle diffusion-adsorption model to describe liquid/solid adsorption kinetics," Revista Mexicana de Ingeniería. Química, vol. 15, pp. 161-173, 2016.

[6] H. Moussout, H. Ahlafi, M. Aazza, and H. Maghat, "Critical of linear and nonlinear equations of pseudo-first order and pseudo-second order kinetic models," Karbala International Journal of Modern Science, vol. 4, no. 2, pp. 244-254, 2018.

[7] J. Huang, F. Liu, and J. Zhang, "Insights into adsorption rate constants and rate laws of preset and arbitrary orders," Separation and Purification Technology, vol. 255, p. 117713, 2021.

[8] S. Azizian, "Kinetic models of sorption: a theoretical analysis," Journal of Colloid and Interface Science, vol. 276, no. 1, pp. 4752, 2004.

[9] J.-P. Simonin, "On the comparison of pseudo-first order and pseudo-second order rate laws in the modeling of adsorption kinetics," Chemical Engineering Journal, vol. 300, pp. 254263, 2016.

[10] A. Özer, "Removal of $\mathrm{Pb}(\mathrm{II})$ ions from aqueous solutions by sulphuric acid-treated wheat bran," Journal of Hazardous Materials, vol. 141, no. 3, pp. 753-761, 2007.

[11] Y. Liu and L. Shen, "A general rate law equation for biosorption," Bio-chemical Engineering Journal, vol. 38, no. 3, pp. 390-394, 2008.

[12] R.-L. Tseng, P.-H. Wu, F.-C. Wu, and R.-S. Juang, “A convenient method to determine kinetic parameters of adsorption processes by nonlinear regression of pseudo-nth-order equation," Chemical Engineering Journal, vol. 237, pp. 153-161, 2014.

[13] W. S. Alencar, E. C. Lima, B. Royer et al., "Application of aqai stalks as biosorbents for the removal of the dye Procion Blue MX-R from aqueous solution," Separation Science and Technology, vol. 47, no. 3, pp. 513-526, 2012.

[14] J. Wang and X. Guo, "Adsorption kinetic models: physical meanings, applications, and solving methods," Journal of Hazardous Materials, vol. 390, article 122156, 2020.

[15] O. O. Aalen and H. K. Gjessing, "Survival models based on the Ornstein-Uhlenbeck process," Lifetime Data Analysis, vol. 10, pp. 407-423, 2009. 
[16] N. Castañeda Leyva, L. Pérez-Hernández, and S. RodríguezNarciso, "Métodos estocásticos en finanzas I," Aportaciones Matemáticas: Comunicaciones SMM, vol. 39, pp. 3-43, 2008.

[17] S. Ditlevsen and P. Lansky, "Estimation of the input parameters in the Ornstein-Uhlenbeck neuronal model," Physical Review E, vol. 71, no. 1, 2005.

[18] S. Ditlevsen and A. Samson, Introduction to Stochastic Models in Biology, Springer-Verlag, 2013.

[19] D.-q. Li, J. Wang, Z.-g. Guo, J. Li, and J. Shuai, "Pectin gels cross-linked by $\mathrm{Ca}^{2+}$ : an efficient material for methylene blue removal," Journal of Molecular Liquids, vol. 238, pp. 36-42, 2017.

[20] J. A. Lozano-Alvarez, J. Jáuregui-Rincón, I. Medina-Ramírez, C. Frausto-Reyes, and R. Salinas-Gutiérrez, "Removal of azo dyes with xanthan," Journal of the Mexican Chemical Society, vol. 63, no. 4, pp. 176-198, 2019.

[21] A. D. Eaton, L. S. Clesceri, E. W. Rice, A. E. Greenberg, and M. A. H. Franson, Standard Methods for the Examination of Water \& Wastewater, American Public Health Association, 21st edition, 2005.

[22] O. Sayrafi, R. Salim, and S. Sayraf, "Removal of cadmium from polluted water using decaying leaves-effects of type of leaves and of concentration of cadmium," Journal of Environmental Science and Health. Part A:790 Environmental Science and Engineering and Toxicology, vol. 31, no. 10, pp. 2503-2513, 1996.

[23] N. Chaiyapo and N. Phewchean, "An application of OrnsteinUhlenbeck process to commodity pricing in Thailand," Advances in Difference Equations, vol. 2017, 2017.

[24] L. Alili, P. Patie, and J. L. Pedersen, "Representations of the first hitting time density of an Ornstein-Uhlenbeck process," Stochastic Models, vol. 21, no. 4, pp. 967-980, 2005.

[25] G. Crini, E. Lichtfouse, L. D. Wilson, and N. Morin-Crini, Adsorption-Oriented Processes Using Conventional and Nonconventional Adsorbents for Wastewater Treatment, Springer International Publishing, 2018.

[26] S. Rodríguez and E. R. Villa, "Stopping times determination for linear degradation processes," Estadística, vol. 63, no. 181, pp. 77-94, 2011.

[27] S. Rodríguez-Narciso and J. A. Christen, "Optimal sequential Bayesian analysis for degradation tests," Lifetime Data Analysis, vol. 22, no. 3, pp. 405-428, 2016.

[28] D. Sprott, Statistical Inference in Science, Springer Verlag, 2000.

[29] R Core Team, R: A Language and Environment for Statistical Computing, R Foundation for Statistical Computing, Vienna, Austria, 2013.

[30] O. A. Attallah, M. A. Al-Ghobashy, M. Nebsen, and M. Y. Salem, "Removal of cationic and anionic dyes from aqueous solution with magnetite/pectin and magnetite/silica/pectin hybrid nanocomposites: kinetic, isotherm and mechanism analysis," RSC Advances, vol. 6, no. 14, pp. 11461-11480, 2016.

[31] J. A. Lozano-Alvarez, V.-F. Marañón Ruiz, J. Jáuregui-Rincón, I. Medina-Ramírez, C. Frausto-Reyes, and R. SalinasGutierrez, "Removal of direct dyes with alginic acid," Journal of the Mexican Chemical Society, vol. 59, no. 3, pp. 215-228, 2015.

[32] M. Barikani, E. Oliaei, H. Seddiqi, and H. Honarkar, "Preparation and application of chitin and its derivatives: a review," Iranian Polymer Journal, vol. 23, pp. 307-326, 2014.
[33] L. Pietrelli, I. Francolini, and A. Piozzi, "Dyes adsorption from aqueous solutions by chitosan," Separation Science and Technology, vol. 50, no. 8, pp. 1101-1107, 2015.

[34] J. A. Lozano-Álvarez, J. Jáuregui-Rincón, G. Mendoza-Díaaz, R. Rodríguez-Vázquez, and C. Frausto-Reyes, "Study of sorption equilibrium of biopolymers alginic acid and xanthan with C.I. disperse yellow 54," Journal of the Mexican Chemical Society, vol. 53, no. 2, pp. 59-70, 2009.

[35] N. R. Singha, M. Mahapatra, M. Karmakar et al., "In situ allocation of a monomer in pectin-g-terpolymer hydrogels and effect of comonomer compositions on superadsorption of metal ions/dyes," ACS Omega, vol. 3, no. 4, pp. 4163-4180, 2018.

[36] L. Largitte and R. Pasquier, "A review of the kinetics adsorption models and their application to the adsorption of lead by an activated carbon," Chemical Engineering Research and Design, vol. 109, pp. 495-504, 2016.

[37] J. A. Lozano-Álvarez, V. F. Marañón Ruiz, J. Jáuregui-Rincón, I. Medina-Ramírez, R. Salinas-Gutiérrez, and C. FraustoReyes, "Remoción de colorantes azo con alginato: relación entre estructura de colorante y eficiencia de remoción," Revista Internacional de Contaminación Ambiental, vol. 35, no. 1, pp. 223-236, 2019.

[38] M. Ferus-Comelo and A. Greaves, "An investigation into direct dye aggregation," Coloration Technology, vol. 118, no. 1, pp. 15-19, 2002.

[39] J. Oakes and S. Dixon, "Physical interactions of dyes in solution - influence of dye structure on aggregation and binding to surfactantsJpolymers," Review of Progress in Coloration and Related Topics, vol. 34, no. 1, pp. 110-847, 2004.

[40] M. Li and G. Buschle-Diller, "Pectin-blended anionic polysaccharide films for cationic contaminant sorption from water," International Journal of Biological Macromolecules, vol. 101, pp. 481-489, 2017.

[41] I. Braccini and S. Pérez, "Molecular basis of $\mathrm{Ca}^{2+}$-induced gelation in alginates and pectins: the egg-box model revisited," Biomacromolecules, vol. 2, no. 4, pp. 1089-1096, 2001.

[42] M.-C. Ralet, V. Dronnet, H. C. Buchholt, and J.-F. Thibault, "Enzymatically and chemically de-esterified lime pectins: characterisation, polyelectrolyte behaviour and calcium binding properties," Carbohydrate Research, vol. 336, no. 2, pp. 117-125, 2001.

[43] M. Clark, Handbook of Textile and Industrial Dyeing, vol. vol. 1 of Woodhead Publishing Series in Textiles, Woodhead Publishing, 2011.

[44] I. Braccini, R. P. Grasso, and S. Pérez, "Conformational and configurational features of acidic polysaccharides and their interactions with calcium ions: a molecular modeling investigation," Carbohydrate Research, vol. 317, no. 1, pp. 119-130, 1999.

[45] J. Lopes da Silva and M. Rao, Pectins: Structure, Functionality, and Uses, CRC Press, 2006.

[46] Z. Chen, Z. Luan, Z. Jia, and X. Li, "On the acid-base stability of Keggin $\mathrm{Al}_{13}$ and $\mathrm{Al}_{30}$ polymers in polyaluminum coagulants," Journal of Materials Science, vol. 44, no. 12, pp. 30983111, 2009.

[47] J. Rowsell and L. F. Nazar, "Speciation and thermal transformation in alumina sols: structures of the polyhydroxyoxoaluminum cluster $\left[\mathrm{Al}_{30} \mathrm{O}_{8}(\mathrm{OH})_{56}(\mathrm{H} 2 \mathrm{O})_{26}\right]^{18+}$ and its $\delta$-Keggin moieté," Journal of the American Chemical Society, vol. 122, no. 15, pp. 3777-3778, 2000. 
[48] A. Caroni, C. de Lima, M. Pereira, and J. Fonseca, "The kinetics of adsorption of tetracycline on chitosan particles," Journal of Colloid and Interface Science, vol. 340, no. 2, pp. 182-191, 2009.

[49] M. Tutt and J. Olt, "Suitability of various plant species for bioethanol production," Agronomy Research, vol. 9, no. 497, pp. 261-267, 2011.

[50] X. Guo, S. Zhang, and X.-q. Shan, "Adsorption of metal ions on lignin," Journal of Hazardous Materials, vol. 151, no. 1, pp. 134-142, 2008.

[51] J. Ralph, C. Lapierre, and W. Boerjan, "Lignin structure and its engineering," Current Opinion in Biotechnology, vol. 56, pp. 240-249, 2019. 